\author{
ARGONNE NATIONAL LABORATORY \\ 9700 South Cass Avenue \\ Argonne, Illinois 60439
}

\title{
TABLES FOR THE EVALUATION OF DECAY CURVES OF A CHEMICAL SPECIES \\ - UNDERGOING SIMULTANEOUS FIRST - AND SECOND-ORDER DECAY
}

by

Klaus H. Schmidt

Chemistry Division

\section{ThE report was prepared NOTICE}

States, nor the Commised as an account of Government sponsored

A. Makes any warranty, not any person acting on behalf of the work. Nelther the Unted racy. completeness, warranty or representation, expressed or implitiong Commission:

of any information, or usefulness of the information contor implied, with respect to the accuprivately ormation, apparatus, method, or procetion contalned in this report, or that the use B. Assued rights; or

use of any infor any liabllitles with respect to the use of or

As used in the apparatus, method, or process disclos damages resulting from the ployee or contractor above, "person acting on behalf of the Com in this report.

such employee or contra commission, or employee of commission" includes any emdisseminates, or providrior of the Commission, or employ contractor, to the extent that with the com or provides access to, any information pursee of such contractor prepares with the Commission, or his employment with mation pursusnt to his employment prepares,

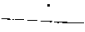

December 1967 


\section{DISCLAIMER}

This report was prepared as an account of work sponsored by an agency of the United States Government. Neither the United States Government nor any agency Thereof, nor any of their employees, makes any warranty, express or implied, or assumes any legal liability or responsibility for the accuracy, completeness, or usefulness of any information, apparatus, product, or process disclosed, or represents that its use would not infringe privately owned rights. Reference herein to any specific commercial product, process, or service by trade name, trademark, manufacturer, or otherwise does not necessarily constitute or imply its endorsement, recommendation, or favoring by the United States Government or any agency thereof. The views and opinions of authors expressed herein do not necessarily state or reflect those of the United States Government or any agency thereof. 


\section{DISCLAIMER}

Portions of this document may be illegible in electronic image products. Images are produced from the best available original document. 
TABLE OF CONTENTS

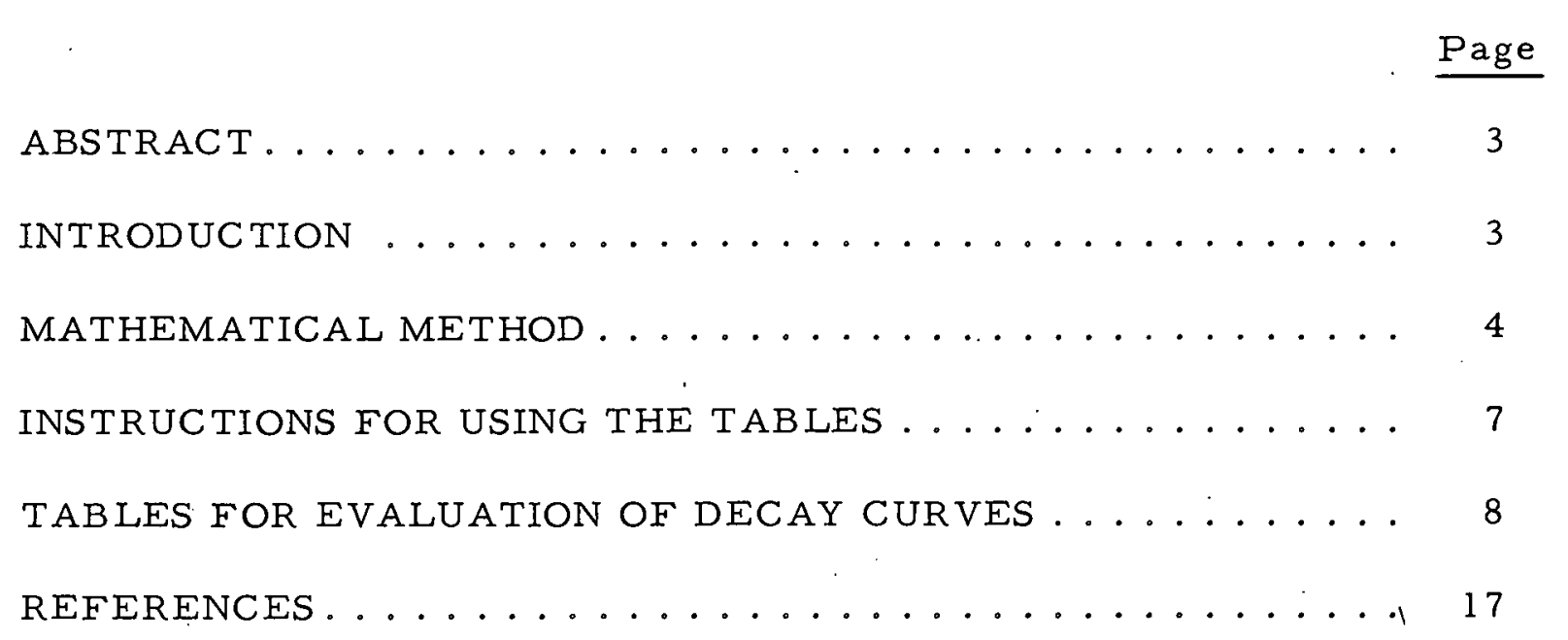




\author{
TABLES FOR THE EVALUATION OF \\ DECAY CURVES OF A CHEMICAL SPECIES \\ UNDERGOING SIMULTANEOUS FIRST - AND \\ SECOND-ORDER DECAY
}

by

Klaus H. Schmidt

\begin{abstract}
Tables have been computed to facilitate the determination of the first- and second-order components of the decay curve of a chemical species from two measured half-lives.
\end{abstract}

\title{
INTRODUCTION
}

In pulse-radiolysis studies, ${ }^{1,2}$ the formation and decay of transient chemical species are directly observed on the oscilloscope. The oscilloscope traces are often used to calculate the rate constant for a first-* or second-order reaction of the chemical species under investigation.

For a pure first- or second-order reaction, the evaluation is usually carried out by plotting the logarithm or the reciprocal, respectively, of the concentration against time and measuring the slope of the resulting straight line. This procedure still works for a predominantly second-order decay with a small first-order component, or vice versa. One then observes a small deviation from the straight line at one end of the curve.

However, if the first- and second-order components are in the same order of magnitude, a more complicated analysis of the decay. curves is required. To facilitate this procedure, the tables described in this paper have been calculated, using an IBM-1620 digital computer. They allow fast calculation of the first- and second-order rate constants from two half-lives and the corresponding initial concentrations, obtained either from one or two different decay curves.

*For the sake of brevity, we shall use the term "first-order reaction" for genuine first-order reactions as well as pseudo first-order reactions. 


\section{MATHEMATICAL METHOD}

The analysis is based on the assumption that the specie $R$ undergoes the reactions

$$
\mathrm{R}+\mathrm{R} \stackrel{\mathrm{k}_{2}}{\longrightarrow} \mathrm{P}
$$

and

$$
\mathrm{R}+\mathrm{S}_{\mathrm{i}} \stackrel{\mathrm{k}_{1}^{(\mathrm{i})}}{\longrightarrow} \mathrm{Q}, \quad \mathrm{i}=1, \ldots, \mathrm{n}
$$

where the $S_{i}$ represent one or more scavengers, $k_{1}^{(i)}$ and $k_{2}$ are secondorder rate constants, and $P$ and $Q$ are products with which $R$ does not react.

The decay of $R$ then follows the differential equation

$$
\frac{d[R]}{d t}=-\left(\sum_{i=1}^{n} k_{1}^{(i)}\left[S_{i}\right]\right)[R]-2 k_{2}[R]^{2}
$$

or, in abbreviated form,

$$
\frac{d c}{d t}=-a c-b c^{2}, \quad c=c_{0} \text { for } t=0 \text {, }
$$

$c_{0}$ being the initial concentration, and

$$
\begin{aligned}
& c=[R], \\
& a=\sum_{i=1}^{n} k_{1}^{(i)}\left[S_{i}\right],
\end{aligned}
$$

and

$$
\mathrm{b}=2 \mathrm{k}_{2} \text {. }
$$

By integration, we obtain

$$
-\int_{c_{0}}^{c} \frac{d c}{a c+b c^{2}}=t
$$

The half-life $T_{1 / 2}$ can now be calculated by setting $c=c_{0} / 2$ : 


$$
T_{1 / 2}=\int_{c_{0} / 2}^{c_{0}} \frac{d c}{c(a+b c)}=\frac{1}{a} \log \frac{b c_{0}+2 a}{b c_{0}+a}
$$

Let $T_{1 / 2}^{(1)}$ and $T_{1 / 2}^{(2)}$ be the half-lives obtained from two decay curves or two parts of the same decay curve with the initial concentrations $c_{0}^{(1)}$ and $c_{0}^{(2)}$, and let

$$
\mathrm{q}_{1}=\frac{\mathrm{bc} \mathrm{c}_{0}^{(1)}}{\mathrm{a}}
$$

and

$$
\mathrm{q}_{2}=\frac{\mathrm{bc}_{0}^{(2)}}{\mathrm{a}}
$$

be the ratios of second- to first-order reaction rates for the two concentrations. Then the ratio of the two half-lives is

$$
f_{t}=\frac{T_{1 / 2}^{(1)}}{T_{1 / 2}^{(2)}}=\frac{\log \frac{q_{1}+2}{q_{1}+1}}{\log \frac{q_{2}+2}{q_{2}+1}}
$$

If we further define.

$$
f_{c}=\frac{c_{0}^{(2)}}{c_{0}^{(1)}}=\frac{q_{2}}{q_{1}}
$$

and chose the numbering so that $f_{c}>1$, we derive, from Eq. 8;

$$
f_{t}=\frac{\log \frac{q_{1}+2}{q_{1}+1}}{\log \frac{f_{c} q_{1}+2}{f_{c} q_{1}+1}} .
$$

For the selected value of $f_{c}$, we can now plot $f_{t}$ against $q_{1}$. From this plot, $q_{1}$ can be determined for any measured value of $f_{t}$. Using Eqs. 6, 7b, and 9 , we then obtain

$$
a=\frac{\log \frac{q_{1}+2}{q_{1}+1}}{T_{1 / 2}^{(1)}}
$$




$$
\mathrm{q}_{2}=\mathrm{f}_{\mathrm{c}} \mathrm{q}_{1}
$$

$$
b c_{0}^{(2)}=q_{2} \frac{\log \frac{q_{2}+2}{q_{2}+1}}{T_{1 / 2}^{(2)}}
$$

With

$$
\phi\left(q_{1}\right)=\log \frac{q_{1}+2}{q_{1}+1}
$$

and

$$
\Psi\left(\mathrm{q}_{2}\right)=\frac{\mathrm{q}_{2}}{2} \log \frac{\mathrm{q}_{2}+2}{\mathrm{q}_{2}+1}
$$

we can write

$$
\begin{aligned}
& a=\frac{\phi\left(q_{1}\right)}{T_{1 / 2}^{(1)}} ; \\
& b c_{0}^{(2)}=\frac{2 \Psi\left(q_{2}\right)}{T_{1 / 2}^{(2)}} .
\end{aligned}
$$

Here a is the first-order rate constant for the combination of all firstorder reactions. The rate constant of the second-order reaction follows from Eqs. $13 \mathrm{~b}$ and $4 \mathrm{~d}$ and is

$$
k_{2}=\frac{\Psi\left(q_{2}\right)}{c_{0}^{(2)} T_{1 / 2}^{(2)}}
$$

If the measured quantity is the optical absorption of the solution, absorptions instead of concentrations can be used in Eq. 9 with a good approximation, if the initial absorption is

$$
\alpha_{0}=\frac{I_{0}-I}{I_{0}}<<1 . *
$$

In this case,

$$
c_{0}=\frac{0.4343 \alpha_{0}}{\epsilon x}
$$

*For $\alpha_{0}<0.1$, the relative error of $f_{c}$ is less than $2 \%$; for $\alpha_{0}<0.05$, it is less than $0.5 \%$. 
where $\epsilon$ is the molar extinction coefficient, and $\mathbf{x}$ is the optical path length in the solution. We then derive, from Eq. 14 ,

$$
\mathrm{k}_{2}=2.303 \frac{\epsilon_{\mathrm{x}}}{\alpha_{0}} \frac{\Psi\left(\mathrm{q}_{2}\right)}{\mathrm{T}_{1 / 2}^{(2)}}
$$

\section{INSTRUCTIONS FOR USING THE TABLES}

For technical reasons, the notation in the tables differs slightly from that in the text.

Notation in the text: $f_{c} \quad f_{t} \quad \phi\left(q_{1}\right) \quad \Psi\left(q_{2}\right) \quad q_{1} \quad q_{1}$

Notation in the tables: $F C$ FT PHI PSI Q1 Q2

The procedure for using the tables is as follows:

1. Find two points on one or two decay curves corresponding to concentrations $c_{0}^{(1)}$ and $c_{0}^{(2)}$ with the ratio

$$
f_{c}=\frac{c_{0}^{(2)}}{c_{0}^{(1)}}=2,2.5,3,4,5, \text { or } 8 \text {. }
$$

2. Measure the half-lives $T_{1 / 2}^{(1)}$ and $T_{1 / 2}^{(2)}$, starting with the above points, and calculate

$$
f_{t}=\frac{T_{1 / 2}^{(1)}}{T_{1 / 2}^{(2)}}
$$

3. Select the table for the chosen value $f_{C}$; and find the values $\phi\left(q_{1}\right)$ and $\Psi\left(q_{2}\right)$ that belong to the obtained value of $f_{t}$.

4. Using Eqs. 13 a and 14 or 17, calculate the rate constants. a and $k_{2}$.

The values $q_{1}$ and $q_{2}$ also listed in the tables give the ratios of secondto first-order reaction rate for the two concentrations $c_{0}^{(1)}$ and $c_{0}^{(2)}$. Values equal to or greater than 100 are printed as 99.999. 
TABLES FOR EVALUATION OF DECAY CURVES

$F C=2 \cdot 0$

$F C=2.0$

\begin{tabular}{|c|c|c|c|c|c|c|c|c|c|}
\hline$=T$ & $\mathrm{PHI}$ & PSI & $Q 1$ & $Q 2$ & $F T$ & PHI & PSI & Q1 & 02 \\
\hline $\begin{array}{l}1.005 \\
1.010 \\
1.015 \\
1.020 \\
1.025\end{array}$ & $\begin{array}{l}.689 \\
.686 \\
.682 \\
.679 \\
.675\end{array}$ & $\begin{array}{r}.004 \\
.009 \\
.014 \\
.018 \\
.023\end{array}$ & $\begin{array}{l}.006 \\
.014 \\
.021 \\
.028 \\
.035\end{array}$ & $\begin{array}{l}.013 \\
.028 \\
.042 \\
.056 \\
.071\end{array}$ & $\begin{array}{l}1.255 \\
1.260 \\
1.265 \\
1.270 \\
1.275\end{array}$ & $\begin{array}{l}.514 \\
.510 \\
.507 \\
.503 \\
.500\end{array}$ & $\begin{array}{r}199 \\
.202 \\
.205 \\
.209 \\
.212\end{array}$ & $\begin{array}{r}.487 \\
.500 \\
.513 \\
.527 \\
.540\end{array}$ & $\begin{array}{r}.974 \\
1.000 \\
1: 027 \\
1: 054 \\
1.081\end{array}$ \\
\hline $\begin{array}{l}.030 \\
.035 \\
.040 \\
.045 \\
.050\end{array}$ & $\begin{array}{l}.672 \\
.668 \\
.665 \\
.661 \\
.658\end{array}$ & $\begin{array}{r}.028 \\
.032 \\
0.037 \\
.041 \\
.046\end{array}$ & $\begin{array}{l}.043 \\
.050 \\
.058 \\
.065 \\
.073\end{array}$ & $\begin{array}{l}.086 \\
.100 \\
.116 \\
.131 \\
.146\end{array}$ & $\begin{array}{l}1 \cdot 280 \\
1.285 \\
1 \cdot 290 \\
1.295 \\
1.300\end{array}$ & $\begin{array}{l}.496 \\
.493 \\
.489 \\
.486 \\
.482\end{array}$ & $\begin{array}{l}.215 \\
.218 \\
.221 \\
.221 \\
.224 \\
.227\end{array}$ & $\begin{array}{r}.554 \\
.568 \\
.583 \\
.597 \\
.612\end{array}$ & $\begin{array}{l}1.109 \\
1: 137 \\
1: 166 \\
1: 195 \\
1.225\end{array}$ \\
\hline $\begin{array}{l}.055 \\
.060 \\
.065 \\
.070 \\
.075\end{array}$ & $\begin{array}{l}.654 \\
.651 \\
.647 \\
.644 \\
.640\end{array}$ & $\begin{array}{l}.050 \\
.054 \\
.059 \\
.063 \\
.067\end{array}$ & $\begin{array}{r}.081 \\
.089 \\
.097 \\
.0105 \\
.113\end{array}$ & $\begin{array}{r}.162 \\
.178 \\
1194 \\
.120 \\
.226\end{array}$ & $\begin{array}{l}1: 305 \\
1: 310 \\
1: 315 \\
1: 320 \\
1 \cdot 325\end{array}$ & $\begin{array}{l}.478 \\
.475 \\
.471 \\
.468 \\
.464\end{array}$ & $\begin{array}{l}.230 \\
.233 \\
.236 \\
.239 \\
.241\end{array}$ & $\begin{array}{l}.627 \\
.642 \\
.658 \\
.673 \\
.689\end{array}$ & $\begin{array}{l}1 \cdot 255 \\
1: 285 \\
1: 316 \\
1: 347 \\
1 \cdot 379\end{array}$ \\
\hline $\begin{array}{l}1.080 \\
1.085 \\
1.090 \\
1.095 \\
1.100\end{array}$ & $\begin{array}{l}.637 \\
.633 \\
.630 \\
.626 \\
.623\end{array}$ & $\begin{array}{l}.071 \\
.075 \\
.080 \\
.084 \\
.088\end{array}$ & 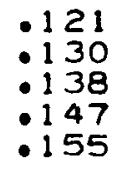 & $\begin{array}{r}.243 \\
.260 \\
.277 \\
.294 \\
.211\end{array}$ & $\begin{array}{l}1 \cdot 330 \\
1: 335 \\
1: 340 \\
1: 345 \\
1 \cdot 350\end{array}$ & $\begin{array}{l}.461 \\
.457 \\
.454 \\
.450 \\
.447\end{array}$ & $\begin{array}{l}.244 \\
.247 \\
.250 \\
.253 \\
.256\end{array}$ & $\begin{array}{l}.705 \\
.722 \\
.738 \\
.755 \\
.772\end{array}$ & $\begin{array}{l}1.411 \\
1.444 \\
1.477 \\
1.511 \\
1.545\end{array}$ \\
\hline 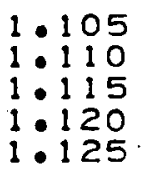 & $\begin{array}{l}.619 \\
.616 \\
.612 \\
.609 \\
.605\end{array}$ & $\begin{array}{r}.092 \\
.096 \\
1100 \\
1104 \\
.108\end{array}$ & $\begin{array}{r}1164 \\
1173 \\
1182 \\
1192 \\
1191 \\
.200\end{array}$ & $\begin{array}{r}.329 \\
.346 \\
.364 \\
.383 \\
.401\end{array}$ & $\begin{array}{l}1 \cdot 355 \\
1: 360 \\
1: 365 \\
1: 370 \\
1 \cdot 375\end{array}$ & $\begin{array}{l}.443 \\
.440 \\
.436 \\
.433 \\
.429\end{array}$ & $\begin{array}{l}.258 \\
.261 \\
.264 \\
.267 \\
.269\end{array}$ & $\begin{array}{l}.790 \\
.808 \\
.826 \\
.844 \\
.863\end{array}$ & $\begin{array}{r}1.580 \\
1.616 \\
1.652 \\
1.688 \\
1.726\end{array}$ \\
\hline $\begin{array}{l}130 \\
135 \\
140 \\
145 \\
150\end{array}$ & $\begin{array}{r}.602 \\
.598 \\
.595 \\
.591 \\
.588\end{array}$ & 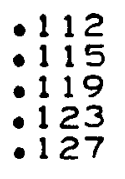 & $\begin{array}{r}.210 \\
.219 \\
.229 \\
.238 \\
.2248\end{array}$ & $\begin{array}{l}.420 \\
.439 \\
.458 \\
.477 \\
.497\end{array}$ & $\begin{array}{l}1.380 \\
1: 385 \\
1: 390 \\
1: 395 \\
1.400\end{array}$ & $\begin{array}{l}.426 \\
.422 \\
.419 \\
.415 \\
.412\end{array}$ & $\begin{array}{r}.272 \\
.275 \\
.277 \\
.280 \\
.282\end{array}$ & $\begin{array}{r}.881 \\
.901 \\
.920 \\
.940 \\
.960\end{array}$ & $\begin{array}{l}1.763 \\
1.802 \\
1.841 \\
1.881 \\
1.921\end{array}$ \\
\hline $\begin{array}{l}16 \\
17 \\
17\end{array}$ & $\begin{array}{r}.584 \\
.581 \\
.577 \\
.574 \\
.570\end{array}$ & 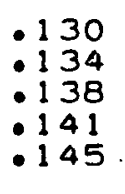 & $\begin{array}{l}.258 \\
.268 \\
.278 \\
.239 \\
.299\end{array}$ & $\begin{array}{l}.517 \\
.537 \\
.557 \\
.578 \\
.599\end{array}$ & $\begin{array}{l}1.405 \\
1.410 \\
1.415 \\
1.420 \\
1.425\end{array}$ & $\begin{array}{l}.408 \\
.405 \\
.401 \\
.398 \\
.394\end{array}$ & $\begin{array}{l}.285 \\
.287 \\
.290 \\
.293 \\
.295\end{array}$ & $\begin{array}{l}.981 \\
1: 002 \\
1.023 \\
1.045 \\
1.067\end{array}$ & $\begin{array}{l}1.963 \\
2.004 \\
2.047 \\
2.090 \\
2.135\end{array}$ \\
\hline $\begin{array}{l}180 \\
185 \\
190 \\
195\end{array}$ & $\begin{array}{r}.567 \\
.563 \\
.560 \\
.556 \\
.553\end{array}$ & $\begin{array}{r}149 \\
1152 \\
1156 \\
159 \\
1163\end{array}$ & $\begin{array}{r}.310 \\
.321 \\
.331 \\
.342 \\
.354\end{array}$ & $\begin{array}{l}.620 \\
.642 \\
.663 \\
.685 \\
.708\end{array}$ & $\begin{array}{l}1.4 \\
1: 4 \\
1: 4 \\
1: 4 \\
1.4\end{array}$ & $\begin{array}{r}.391 \\
: 387 \\
.383 \\
.380 \\
.376\end{array}$ & $\begin{array}{l}\cdot 298 \\
: 300 \\
\cdot 302 \\
: 305 \\
: 307\end{array}$ & $\begin{array}{l}1.089 \\
1: 1112 \\
1: 136 \\
1: 159 \\
1: 183\end{array}$ & $\begin{array}{l}2 \cdot 179 \\
2 \cdot 225 \\
2 \cdot 272 \\
2 \cdot 319 \\
2 \cdot 367\end{array}$ \\
\hline $\begin{array}{l}205 \\
210 \\
215 \\
220 \\
225\end{array}$ & $\begin{array}{l}.549 \\
.545 \\
.542 \\
.538 \\
.535\end{array}$ & $\begin{array}{l}.166 \\
.170 \\
1173 \\
1176 \\
1180\end{array}$ & $\begin{array}{l}.365 \\
.376 \\
.388 \\
.400 \\
.412\end{array}$ & $\begin{array}{l}.730 \\
.753 \\
.776 \\
.800 \\
.824\end{array}$ & $\begin{array}{l}1.455 \\
1.460 \\
1.465 \\
1.470 \\
1.475\end{array}$ & $\begin{array}{l}.373 \\
.369 \\
.366 \\
.362 \\
.359\end{array}$ & $\begin{array}{r}.310 \\
.312 \\
.314 \\
.317 \\
.319\end{array}$ & $\begin{array}{l}1.208 \\
1.233 \\
1.259 \\
1.285 \\
1.311\end{array}$ & $\begin{array}{l}2.417 \\
2.467 \\
2.518 \\
2.570 \\
2.623\end{array}$ \\
\hline 24 & $\begin{array}{r}.531 \\
.528 \\
.524 \\
.521 \\
.517\end{array}$ & $\begin{array}{r}183 \\
1186 \\
1190 \\
1193 \\
1196\end{array}$ & $\begin{array}{l}.424 \\
.436 \\
.448 \\
.461 \\
.474\end{array}$ & $\begin{array}{r}.848 \\
.872 \\
.897 \\
.923 \\
.948\end{array}$ & $\begin{array}{l}1.4 \\
1.4 \\
1.4 \\
1.4\end{array}$ & $\begin{array}{l}.355 \\
.352 \\
.348 \\
.345 \\
.341\end{array}$ & $\begin{array}{r}.322 \\
.324 \\
.326 \\
.328 \\
.331\end{array}$ & $\begin{array}{l}1 \cdot 338 \\
1: 366 \\
1: 394 \\
1: 423 \\
1.452\end{array}$ & $\begin{array}{l}2.677 \\
2 \cdot 732 \\
2.789 \\
2.846 \\
2.905\end{array}$ \\
\hline
\end{tabular}


$F C=2.0$

$F C=2.5$

\begin{tabular}{|c|c|c|c|c|c|c|c|c|c|}
\hline$F T$ & PHI & PSI & Q1 & Q2 & $F T$ & PHI & PSI & Q1 & Q2 \\
\hline $\begin{array}{l}1 \cdot 51 \\
1: 52 \\
1: 53 \\
1: 54 \\
1.55\end{array}$ & $\begin{array}{r}.334 \\
: 328 \\
: 321 \\
: 314 \\
: 307\end{array}$ & $\begin{array}{r}.335 \\
: 340 \\
: 344 \\
: 348 \\
: 353\end{array}$ & $\begin{array}{l}1.513 \\
1.576 \\
1.641 \\
1.710 \\
1.781\end{array}$ & $\begin{array}{l}3.026 \\
3.152 \\
3.283 \\
3.420 \\
3.563\end{array}$ & $\begin{array}{l}1.01 \\
1: 02 \\
1: 03 \\
1: 04 \\
1: 05\end{array}$ & $\begin{array}{l}.688 \\
.683 \\
.679 \\
.674 \\
.669\end{array}$ & $\begin{array}{l}.007 \\
.015 \\
0 \\
0223 \\
0 \\
030 \\
0 \\
038\end{array}$ & $\begin{array}{l}.009 \\
.018 \\
.028 \\
.038 \\
.048\end{array}$ & $\begin{array}{r}.023 \\
.046 \\
.070 \\
.095 \\
. \\
0\end{array}$ \\
\hline $\begin{array}{l}1.56 \\
1.57 \\
1.58 \\
1.59 \\
1.60\end{array}$ & $\begin{array}{r}.300 \\
.293 \\
.286 \\
.279 \\
.272\end{array}$ & $\begin{array}{l}.357 \\
.361 \\
.365 \\
.369 \\
.373\end{array}$ & $\begin{array}{l}1.856 \\
1.935 \\
2.017 \\
2.104 \\
2.194\end{array}$ & $\begin{array}{l}3.713 \\
3.870 \\
4.035 \\
4.208 \\
4.389\end{array}$ & $\begin{array}{l}1.06 \\
1.07 \\
1.08 \\
1.09 \\
1.10\end{array}$ & $\begin{array}{l}.665 \\
.660 \\
.656 \\
.651 \\
.646\end{array}$ & $\begin{array}{l}.045 \\
.052 \\
.059 \\
.066 \\
.073\end{array}$ & $\begin{array}{l}.058 \\
.068 \\
.078 \\
.089 \\
.099\end{array}$ & 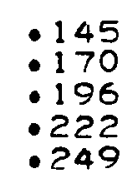 \\
\hline $\begin{array}{l}1.61 \\
1.62 \\
1.63 \\
1.64 \\
1.65\end{array}$ & $\begin{array}{r}.265 \\
.258 \\
.251 \\
.244 \\
.237\end{array}$ & $\begin{array}{r}\cdot 377 \\
\cdot 381 \\
\cdot 385 \\
\cdot 389 \\
\cdot 392\end{array}$ & $\begin{array}{l}2 \cdot 290 \\
2.390 \\
2.496 \\
2.608 \\
2.726\end{array}$ & $\begin{array}{l}4.580 \\
4.781 \\
4.993 \\
5.216 \\
5.453\end{array}$ & 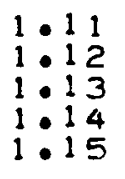 & $\begin{array}{l}.642 \\
.637 \\
.632 \\
.627 \\
.623\end{array}$ & $\begin{array}{r}.080 \\
.086 \\
.093 \\
.099 \\
.105\end{array}$ & 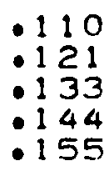 & $\begin{array}{r}.276 \\
.304 \\
.332 \\
.361 \\
.389\end{array}$ \\
\hline $\begin{array}{l}1.66 \\
1.67 \\
1.68 \\
1.69 \\
1.70\end{array}$ & $\begin{array}{r}.230 \\
.2 \\
.223 \\
.217 \\
.210 \\
.203\end{array}$ & $\begin{array}{l}.396 \\
.400 \\
.403 \\
.407 \\
.410\end{array}$ & $\begin{array}{l}2.852 \\
2.984 \\
3.126 \\
3.276 \\
3.437\end{array}$ & $\begin{array}{l}5.704 \\
5.969 \\
6.252 \\
6.553 \\
6.874\end{array}$ & $\begin{array}{l}1: 16 \\
1: 17 \\
1: 18 \\
1: 19 \\
1: 20\end{array}$ & $\begin{array}{r}.618 \\
.613 \\
.609 \\
.604 \\
.599\end{array}$ & 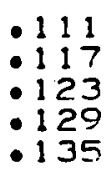 & $\begin{array}{r}167 \\
.179 \\
.191 \\
.204 \\
.216\end{array}$ & $\begin{array}{r}.419 \\
.449 \\
.479 \\
.510 \\
.542\end{array}$ \\
\hline $\begin{array}{l}1: 71 \\
1: 72 \\
1: 73 \\
1: 74 \\
1: 75\end{array}$ & 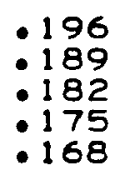 & $\begin{array}{r}.414 \\
.417 \\
.421 \\
.424 \\
.427\end{array}$ & $\begin{array}{l}3 \cdot 608 \\
3 \cdot 792 \\
3 \cdot 990 \\
4 \cdot 203 \\
4.433\end{array}$ & $\begin{array}{l}7.217 \\
7.585 \\
7.981 \\
8.407 \\
8.867\end{array}$ & $\begin{array}{l}1.21 \\
1 \cdot 22 \\
1: 23 \\
1.224 \\
1.25\end{array}$ & $\begin{array}{r}.595 \\
.590 \\
.585 \\
.581 \\
.570\end{array}$ & $\begin{array}{l}.141 \\
: 146 \\
: 152 \\
: 157 \\
.163\end{array}$ & $\begin{array}{r}.229 \\
.242 \\
.255 \\
.269 \\
.282\end{array}$ & $\begin{array}{l}.573 \\
.606 \\
.639 \\
.673 \\
.707\end{array}$ \\
\hline $\begin{array}{l}1: 76 \\
1: 77 \\
1: 78 \\
1: 79 \\
1: 80\end{array}$ & $\begin{array}{r}162 \\
: 155 \\
1 \\
: 148 \\
: 141 \\
: 134\end{array}$ & $\begin{array}{r}.431 \\
.434 \\
.437 \\
.440 \\
.444\end{array}$ & $\begin{array}{l}4.682 \\
4.954 \\
5 \cdot 249 \\
5 \cdot 573 \\
5.930\end{array}$ & $\begin{array}{r}9.365 \\
9.908 \\
10.499 \\
11.147 \\
11.860\end{array}$ & $\begin{array}{l}1.26 \\
1: 27 \\
1: 28 \\
1: 29 \\
1: 30\end{array}$ & $\begin{array}{l}.571 \\
.566 \\
.562 \\
.557 \\
.552\end{array}$ & $\begin{array}{r}168 \\
: 173 \\
1178 \\
1183 \\
\cdot 188\end{array}$ & $\begin{array}{r}.296 \\
.310 \\
.325 \\
: 339 \\
\cdot 354\end{array}$ & $\begin{array}{r}.741 \\
.777 \\
.813 \\
.849 \\
.886\end{array}$ \\
\hline $\begin{array}{l}1.81 \\
1.82 \\
1: 83 \\
1.84 \\
1.85\end{array}$ & 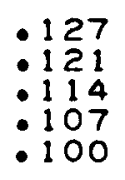 & $\begin{array}{l}.447 \\
.450 \\
.453 \\
.456 \\
.459\end{array}$ & $\begin{array}{l}6.324 \\
6.762 \\
7.251 \\
7.802 \\
8.426\end{array}$ & $\begin{array}{l}12.648 \\
13.524 \\
14.503 \\
15.604 \\
16.853\end{array}$ & $\begin{array}{l}1 \cdot 31 \\
1: 32 \\
1: 33 \\
1: 34 \\
1: 35\end{array}$ & $\begin{array}{r}.548 \\
.543 \\
.538 \\
.533 \\
.529\end{array}$ & $\begin{array}{r}.193 \\
: 198 \\
.203 \\
.207 \\
.212\end{array}$ & $\begin{array}{r}.369 \\
.385 \\
.401 \\
.417 \\
.433\end{array}$ & $\begin{array}{r}.924 \\
.963 \\
1.002 \\
1.042 \\
1.083\end{array}$ \\
\hline $\begin{array}{l}1.86 \\
1.87 \\
1.88 \\
1.89 \\
1.90\end{array}$ & $\begin{array}{r}.094 \\
.087 \\
.080 \\
.073 \\
.067\end{array}$ & $\begin{array}{l}.462 \\
.465 \\
.467 \\
.470 \\
.473\end{array}$ & $\begin{array}{r}9.140 \\
9.963 \\
10.924 \\
12.060 \\
13.423\end{array}$ & $\begin{array}{l}18 \cdot 280 \\
19.926 \\
21.849 \\
24.120 \\
26.846\end{array}$ & $\begin{array}{l}1: 36 \\
1: 37 \\
1: 38 \\
1: 39 \\
1: 40\end{array}$ & $\begin{array}{r}.524 \\
.519 \\
.515 \\
.510 \\
.505\end{array}$ & $\begin{array}{l}.216 \\
.221 \\
.225 \\
.230 \\
.234\end{array}$ & $\begin{array}{r}.449 \\
.466 \\
.483 \\
.501 \\
.519\end{array}$ & $\begin{array}{l}1 \cdot 124 \\
1 \cdot 166 \\
1 \cdot 209 \\
1 \cdot 253 \\
1 \cdot 298\end{array}$ \\
\hline $\begin{array}{l}.91 \\
1.92 \\
1.93 \\
1.94\end{array}$ & $\begin{array}{r}.060 \\
.053 \\
.046 \\
.040 \\
.033\end{array}$ & $\begin{array}{r}.476 \\
.479 \\
.481 \\
.484 \\
.487\end{array}$ & $\begin{array}{l}15.089 \\
17.171 \\
19.850 \\
23.420 \\
28.419\end{array}$ & $\begin{array}{l}30 \cdot 178 \\
34 \cdot 343 \\
39 \cdot 700 \\
46 \cdot 841 \\
56 \cdot 8.38\end{array}$ & $\begin{array}{l}1.41 \\
1.42 \\
1.43 \\
1.44 \\
1.45\end{array}$ & $\begin{array}{l}.501 \\
.496 \\
.491 \\
.486 \\
.482\end{array}$ & $\begin{array}{l}.238 \\
.242 \\
.247 \\
.251 \\
.255\end{array}$ & $\begin{array}{r}.537 \\
.556 \\
.574 \\
.594 \\
.513\end{array}$ & $\begin{array}{l}1.343 \\
1.390 \\
1.437 \\
1.485 \\
1.534\end{array}$ \\
\hline $\begin{array}{l}1.96 \\
1: 97 \\
1: 98 \\
1.99\end{array}$ & $\begin{array}{l}.026 \\
.020 \\
.013 \\
.006 \\
.000\end{array}$ & $\begin{array}{r}.489 \\
.492 \\
.494 \\
.497 \\
.500\end{array}$ & $\begin{array}{l}35.923 \\
48.416 \\
73.430 \\
99.999 \\
99.999\end{array}$ & $\begin{array}{l}71.847 \\
96.833 \\
99.999 \\
99.999 \\
99.999\end{array}$ & $\begin{array}{l}1.46 \\
1.47 \\
1.48 \\
1.49 \\
1.50\end{array}$ & $\begin{array}{l}.477 \\
.472 \\
.458 \\
.463 \\
.458\end{array}$ & $\begin{array}{r}.259 \\
.263 \\
.266 \\
.270 \\
.274\end{array}$ & $\begin{array}{l}.633 \\
.654 \\
.675 \\
.696 \\
.718\end{array}$ & $\begin{array}{l}1.584 \\
1.636 \\
1.688 \\
1.741 \\
1.795\end{array}$ \\
\hline
\end{tabular}


$F C=2.5$

$F C=3.0$

\begin{tabular}{|c|c|c|c|c|c|c|c|c|c|}
\hline FT & $\mathrm{PHI}$ & PSI & $Q_{1}$ & $Q 2$ & $F T$ & PHI & PSI & $Q_{1}$ & Q2 \\
\hline $\begin{array}{l}1.52 \\
1.54 \\
1.56 \\
1.58 \\
1.60\end{array}$ & $\begin{array}{r}.449 \\
.439 \\
.430 \\
.421 \\
.411\end{array}$ & $\begin{array}{r}.281 \\
: 289 \\
: 296 \\
: 303 \\
\cdot 309\end{array}$ & $\begin{array}{r}.763 \\
.809 \\
.858 \\
.909 \\
.962\end{array}$ & $\begin{array}{l}1.907 \\
2.024 \\
2.146 \\
2.274 \\
2.407\end{array}$ & $\begin{array}{l}1.02 \\
1.04 \\
1.06 \\
1.08 \\
1.10\end{array}$ & $\begin{array}{l}.686 \\
.679 \\
.672 \\
.665 \\
.658\end{array}$ & $\begin{array}{l}.014 \\
.027 \\
.040 \\
.053 \\
.065\end{array}$ & $\begin{array}{r}.014 \\
.028 \\
.043 \\
.058 \\
.073\end{array}$ & $\begin{array}{r}.042 \\
.085 \\
.129 \\
.174 \\
.220\end{array}$ \\
\hline $\begin{array}{l}1.62 \\
1.64 \\
1.66 \\
1.68 \\
1.70\end{array}$ & $\begin{array}{r}.402 \\
.393 \\
.383 \\
.374 \\
.364\end{array}$ & $\begin{array}{r}.316 \\
.322 \\
.328 \\
: 334 \\
.340\end{array}$ & $\begin{array}{l}1.018 \\
1: 077 \\
1 \cdot 138 \\
1.202 \\
1.270\end{array}$ & $\begin{array}{l}2.546 \\
2.692 \\
2.846 \\
3.006 \\
3.175\end{array}$ & 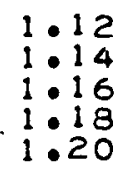 & $\begin{array}{l}.651 \\
.644 \\
.637 \\
.630 \\
.623\end{array}$ & $\begin{array}{r}.077 \\
.089 \\
0100 \\
1111 \\
11 \\
.11\end{array}$ & $\begin{array}{l}.089 \\
.1105 \\
11 \\
1121 \\
.138 \\
.156\end{array}$ & $\begin{array}{l}.267 \\
.316 \\
.365 \\
.416 \\
.468\end{array}$ \\
\hline $\begin{array}{l}1: 72 \\
1: 74 \\
1: 76 \\
1: 78 \\
1.80\end{array}$ & $\begin{array}{r}.355 \\
.346 \\
.336 \\
.327 \\
\cdot 318\end{array}$ & $\begin{array}{r}.346 \\
.352 \\
.357 \\
.363 \\
.368\end{array}$ & $\begin{array}{l}1.341 \\
1.416 \\
1.495 \\
1.578 \\
1.567\end{array}$ & $\begin{array}{l}3.353 \\
3.540 \\
3.738 \\
3.947 \\
4.168\end{array}$ & $\begin{array}{l}1.22 \\
1.24 \\
1.26 \\
1.23 \\
1.30\end{array}$ & $\begin{array}{l}.616 \\
.609 \\
.602 \\
.595 \\
.587\end{array}$ & $\begin{array}{l}131 \\
1141 \\
1151 \\
1160 \\
1 \\
169\end{array}$ & $\begin{array}{r}.173 \\
.192 \\
.210 \\
.229 \\
.249\end{array}$ & $\begin{array}{r}.521 \\
.576 \\
.632 \\
.589 \\
.748\end{array}$ \\
\hline $\begin{array}{l}1.82 \\
1.84 \\
1.86 \\
1.88 \\
1.90\end{array}$ & $\begin{array}{r}.309 \\
.299 \\
.290 \\
.281 \\
.272\end{array}$ & $\begin{array}{r}\cdot 373 \\
\cdot 378 \\
\cdot 383 \\
\cdot 388 \\
\cdot 393\end{array}$ & $\begin{array}{l}1.760 \\
1.860 \\
1.965 \\
2.078 \\
2.198\end{array}$ & $\begin{array}{l}4.401 \\
4.650 \\
4.914 \\
5.195 \\
5.495\end{array}$ & $\begin{array}{l}1: 32 \\
1: 34 \\
1: 36 \\
1: 38 \\
1: 40\end{array}$ & $\begin{array}{l}.580 \\
.573 \\
.566 \\
.559 \\
.552\end{array}$ & $\begin{array}{r}177 \\
1186 \\
1194 \\
.202 \\
.210\end{array}$ & $\begin{array}{r}.269 \\
.290 \\
.311 \\
.333 \\
.355\end{array}$ & $\begin{array}{r}.808 \\
.870 \\
.934 \\
1.999 \\
1.066\end{array}$ \\
\hline $\begin{array}{l}1.92 \\
1: 94 \\
1: 96 \\
1: 98 \\
2.00\end{array}$ & $\begin{array}{r}.262 \\
.253 \\
.244 \\
.235 \\
.226\end{array}$ & $\begin{array}{r}.398 \\
.402 \\
.407 \\
.411 \\
.415\end{array}$ & $\begin{array}{l}2.326 \\
2.464 \\
2.612 \\
2.771 \\
2.943\end{array}$ & $\begin{array}{l}5.816 \\
6.160 \\
6.530 \\
6.928 \\
7.358\end{array}$ & $\begin{array}{l}1.42 \\
1.44 \\
1.46 \\
1.48 \\
1.50\end{array}$ & $\begin{array}{l}.545 \\
.538 \\
.531 \\
.524 \\
.517\end{array}$ & $\begin{array}{r}.217 \\
.225 \\
.232 \\
.239 \\
.246\end{array}$ & $\begin{array}{l}.378 \\
.401 \\
.425 \\
.450 \\
.476\end{array}$ & $\begin{array}{l}1 \cdot 134 \\
1: 205 \\
1: 277 \\
1: 352 \\
1.428\end{array}$ \\
\hline $\begin{array}{l}2.02 \\
2.04 \\
2.06 \\
2.08 \\
2.10\end{array}$ & $\begin{array}{r}.216 \\
.207 \\
.198 \\
1189 \\
.180\end{array}$ & $\begin{array}{r}.419 \\
.424 \\
.428 \\
.432 \\
.435\end{array}$ & $\begin{array}{l}3.130 \\
3.333 \\
3.554 \\
3.797 \\
4.064\end{array}$ & $\begin{array}{r}7.825 \\
8 \cdot 332 \\
8 \cdot 886 \\
9.492 \\
10.160\end{array}$ & $\begin{array}{l}1.52 \\
1.54 \\
1.56 \\
1.58 \\
1.60\end{array}$ & $\begin{array}{l}.510 \\
.503 \\
.496 \\
.488 \\
.481\end{array}$ & $\begin{array}{l}.252 \\
.259 \\
.265 \\
.271 \\
.277\end{array}$ & $\begin{array}{r}.502 \\
.529 \\
.557 \\
.585 \\
.615\end{array}$ & $\begin{array}{l}1.507 \\
1.588 \\
1.671 \\
1.756 \\
1.845\end{array}$ \\
\hline $\begin{array}{l}2: 12 . \\
2: 14 \\
2: 16 \\
2: 18 \\
2: 20\end{array}$ & 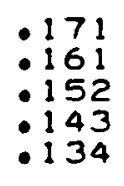 & $\begin{array}{r}.439 \\
.443 \\
.447 \\
.450 \\
.454\end{array}$ & $\begin{array}{l}4 \cdot 359 \\
4.687 \\
5.054 \\
5.466 \\
5.934\end{array}$ & $\begin{array}{l}10.898 \\
11.718 \\
12.635 \\
13.667 \\
14.836\end{array}$ & $\begin{array}{l}1.62 \\
1.64 \\
1.66 \\
1.68 \\
1.70\end{array}$ & $\begin{array}{l}.474 \\
.467 \\
.460 \\
.453 \\
.446\end{array}$ & $\begin{array}{r}.283 \\
.289 \\
.295 \\
.300 \\
.305\end{array}$ & $\begin{array}{l}.645 \\
.676 \\
.708 \\
.741 \\
.776\end{array}$ & $\begin{array}{l}1 \cdot 9.35 \\
2 \cdot 029 \\
2 \cdot 125 \\
2 \cdot 225 \\
2 \cdot 328\end{array}$ \\
\hline $\begin{array}{l}2 \cdot 22 \\
2: 24 \\
2 \cdot 26 \\
2 \cdot 28 \\
2 \cdot 30\end{array}$ & $\begin{array}{l}.125 \\
.116 \\
.107 \\
.098 \\
.089\end{array}$ & $\begin{array}{l}.457 \\
.461 \\
.464 \\
.467 \\
.470\end{array}$ & $\begin{array}{l}6.469 \\
7.086 \\
7.807 \\
8.658 \\
9.680\end{array}$ & $\begin{array}{l}16.173 \\
17.716 \\
19.517 \\
21.646 \\
24.201\end{array}$ & $\begin{array}{l}1.72 \\
1: 74 \\
1: 76 \\
1.78 \\
1.80\end{array}$ & $\begin{array}{r}.439 \\
.432 \\
.425 \\
.418 \\
.411\end{array}$ & $\begin{array}{r}.311 \\
: 316 \\
.321 \\
.321 \\
.325 \\
.330\end{array}$ & $\begin{array}{l}.811 \\
.847 \\
.885 \\
.924 \\
.964\end{array}$ & $\begin{array}{l}2.433 \\
2.543 \\
2.556 \\
2.772 \\
2.893\end{array}$ \\
\hline $\begin{array}{l}2 \cdot 32 \\
2: 34 \\
2 \cdot 36 \\
2: 38 \\
2 \cdot 40\end{array}$ & $\begin{array}{l}.080 \\
.071 \\
.062 \\
.053 \\
.044\end{array}$ & $\begin{array}{l}.474 \\
.477 \\
.480 \\
.483 \\
.486\end{array}$ & $\begin{array}{l}10.929 \\
12.491 \\
14.498 \\
17.176 \\
20.924\end{array}$ & $\begin{array}{l}27 \cdot 324 \\
31 \cdot 228 \\
36 \cdot 246 \\
42 \cdot 941 \\
52 \cdot 310\end{array}$ & $\begin{array}{l}1.82 \\
1.84 \\
1.86 \\
1.88 \\
1.90\end{array}$ & $\begin{array}{r}.404 \\
.397 \\
.390 \\
.393 \\
.376\end{array}$ & $\begin{array}{r}.335 \\
.339 \\
.344 \\
.348 \\
\cdot 353\end{array}$ & $\begin{array}{l}1.006 \\
1.049 \\
1.093 \\
1.140 \\
1.188\end{array}$ & $\begin{array}{l}3.018 \\
3.147 \\
3.281 \\
3.420 \\
3.564\end{array}$ \\
\hline $\begin{array}{l}2: 4 \\
2: 4 \\
2: 4 \\
2: 4 \\
2: 5\end{array}$ & $\begin{array}{r}.035 \\
.026 \\
.017 \\
0.008 \\
.000\end{array}$ & $\begin{array}{l}.488 \\
.491 \\
.494 \\
.497 \\
.500\end{array}$ & $\begin{array}{l}26 \cdot 548 \\
35 \cdot 916 \\
54 \cdot 641 \\
99 \cdot 999 \\
99 \cdot 999\end{array}$ & $\begin{array}{l}66 \cdot 371 \\
89 \cdot 791 \\
99.999 \\
99 \cdot 999 \\
99.999\end{array}$ & $\begin{array}{l}1.92 \\
1.94 \\
1.96 \\
1.98 \\
2.00\end{array}$ & $\begin{array}{l}.369 \\
.362 \\
.355 \\
.343 \\
.341\end{array}$ & $\begin{array}{l}.357 \\
.361 \\
.365 \\
.369 \\
.373\end{array}$ & $\begin{array}{l}1.238 \\
1.289 \\
1.343 \\
1.399 \\
1.457\end{array}$ & $\begin{array}{l}3.714 \\
3.869 \\
4.030 \\
4.197 \\
4.372\end{array}$ \\
\hline
\end{tabular}


$F C=3.0$

$F C=4.0$

\begin{tabular}{|c|c|c|c|c|c|c|c|c|c|}
\hline$F T$ & $\mathrm{PHI}$ & PS I & Q1 & Q2 & $F T$ & PHI & PSI & Q1 & $Q 2$ \\
\hline $\begin{array}{l}04 \\
06 \\
08 \\
10\end{array}$ & $\begin{array}{r}.334 \\
-327 \\
: 320 \\
: 313 \\
\cdot 306\end{array}$ & $\begin{array}{r}\cdot 376 \\
: 380 \\
: 384 \\
: 387 \\
: 391\end{array}$ & $\begin{array}{l}1.517 \\
1.581 \\
1.646 \\
1.715 \\
1.787\end{array}$ & $\begin{array}{l}4 \cdot 553 \\
4: 743 \\
4 \cdot 940 \\
5 \cdot 146 \\
5 \cdot 361\end{array}$ & $\begin{array}{l}1.02 \\
1.04 \\
1.06 \\
1.08 \\
1.010\end{array}$ & $\begin{array}{l}.688 \\
.683 \\
.679 \\
.674 \\
.669\end{array}$ & $\begin{array}{r}.012 \\
0024 \\
0 \\
036 \\
.047 \\
.058\end{array}$ & $\begin{array}{r}.009 \\
.018 \\
.028 \\
.038 \\
.048\end{array}$ & 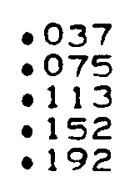 \\
\hline $\begin{array}{l}12 \\
14 \\
16 \\
18 \\
20\end{array}$ & $\begin{array}{r}.299 \\
.292 \\
.285 \\
.278 \\
.271\end{array}$ & $\begin{array}{r}.394 \\
.398 \\
.401 \\
.404 \\
.407\end{array}$ & $\begin{array}{l}1.862 \\
1: 941 \\
2: 023 \\
2: 109 \\
2 \cdot 200\end{array}$ & $\begin{array}{l}5 \cdot 587 \\
5 \cdot 823 \\
6 \cdot 070 \\
6 \cdot 329 \\
6 \cdot 601\end{array}$ & 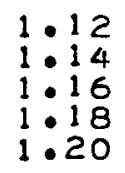 & $\begin{array}{l}.665 \\
.660 \\
.655 \\
.651 \\
.646\end{array}$ & $\begin{array}{l}.069 \\
.079 \\
.089 \\
.098 \\
.107\end{array}$ & $\begin{array}{r}.058 \\
.068 \\
.078 \\
.078 \\
.089 \\
.100\end{array}$ & $\begin{array}{l}.232 \\
.273 \\
: 315 \\
.357 \\
.400\end{array}$ \\
\hline $\begin{array}{l}22 \\
24 \\
26 \\
28 \\
30\end{array}$ & $\begin{array}{r}.264 \\
.258 \\
.251 \\
.244 \\
.237\end{array}$ & $\begin{array}{r}.411 \\
.414 \\
.417 \\
.420 \\
.423\end{array}$ & $\begin{array}{l}2 \cdot 296 \\
2 \cdot 396 \\
2 \cdot 502 \\
2 \cdot 614 \\
2.733\end{array}$ & $\begin{array}{l}6.888 \\
7.190 \\
7.508 \\
7.843 \\
8.199\end{array}$ & $\begin{array}{l}1 \cdot 22 \\
1: 24 \\
1: 26 \\
1: 28 \\
1: 28\end{array}$ & $\begin{array}{l}.641 \\
.637 \\
.632 \\
.627 \\
.623\end{array}$ & $\begin{array}{l}.116 \\
: 1125 \\
: 133 \\
: 142 \\
: 150 .\end{array}$ & $\begin{array}{l}.111 \\
.1122 \\
.133 \\
.1144 \\
.156\end{array}$ & $\begin{array}{l}.444 \\
.488 \\
.533 \\
.579 \\
.626\end{array}$ \\
\hline $\begin{array}{l}32 \\
34 \\
36 \\
38 \\
40\end{array}$ & $\begin{array}{r}.230 \\
.223 \\
.216 \\
.209 \\
.202\end{array}$ & $\begin{array}{r}.425 \\
.428 \\
.431 \\
.434 \\
.436\end{array}$ & $\begin{array}{l}2.858 \\
2.991 \\
3.132 \\
3.283 \\
3.444\end{array}$ & $\begin{array}{r}8 \cdot 575 \\
8 \cdot 974 \\
9 \cdot 398 \\
9 \cdot 850 \\
10.332\end{array}$ & $\begin{array}{l}1: 32 \\
1: 34 \\
1: 36 \\
1: 38 \\
1: 40\end{array}$ & $\begin{array}{l}.618 \\
.613 \\
.608 \\
.604 \\
.599\end{array}$ & $\begin{array}{r}157 \\
: 155 \\
: 172 \\
1179 \\
1186\end{array}$ & $\begin{array}{r}.168 \\
.180 \\
.192 \\
.205 \\
.217\end{array}$ & $\begin{array}{l}.673 \\
.721 \\
.770 \\
.820 \\
.870\end{array}$ \\
\hline $\begin{array}{l}4 \\
6 \\
8 \\
0\end{array}$ & $\begin{array}{r}196 \\
1189 \\
: 182 \\
: 175 \\
: 168\end{array}$ & $\begin{array}{l}.439 \\
.442 \\
.444 \\
.447 \\
.449\end{array}$ & $\begin{array}{l}3.615 \\
3.800 \\
3.997 \\
4.210 \\
4.440\end{array}$ & $\begin{array}{l}10.847 \\
11.400 \\
11.993 \\
12.632 \\
13.322\end{array}$ & $\begin{array}{l}1.42 \\
1.44 \\
1: 46 \\
1: 48 \\
1.50\end{array}$ & $\begin{array}{r}.594 \\
.590 \\
.585 \\
.580 \\
.575\end{array}$ & $\begin{array}{r}.193 \\
.199 \\
. \\
.205 \\
.212 \\
.218\end{array}$ & $\begin{array}{r}.230 \\
.243 \\
.256 \\
.270 \\
.284\end{array}$ & $\begin{array}{l}.922 \\
.974 \\
1.027 \\
1: 081 \\
1.136\end{array}$ \\
\hline & $\begin{array}{r}161 \\
: 155 \\
: 148 \\
: 141 \\
: 134\end{array}$ & $\begin{array}{l}.451 \\
.454 \\
.456 \\
.459 \\
.461\end{array}$ & $\begin{array}{l}4.690 \\
4.961 \\
5.257 \\
5.581 \\
5.9 .37\end{array}$ & $\begin{array}{l}14.071 \\
14.884 \\
15.771 \\
16.743 \\
17.813\end{array}$ & $\begin{array}{l}1.52 \\
1.54 \\
1.56 \\
1.58 \\
1.60\end{array}$ & $\begin{array}{l}.571 \\
.566 \\
.561 \\
.557 \\
.552\end{array}$ & $\begin{array}{r}.224 \\
.229 \\
.235 \\
.240 \\
.246\end{array}$ & $\begin{array}{r}.298 \\
: 312 \\
\because 326 \\
.341 \\
.356\end{array}$ & $\begin{array}{l}1.192 \\
1: 249 \\
1: 307 \\
1: 365 \\
1.425\end{array}$ \\
\hline & 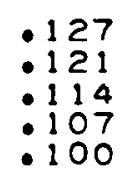 & $\begin{array}{l}.463 \\
.465 \\
.467 \\
.469 \\
.472\end{array}$ & $\begin{array}{l}6.331 \\
6.770 \\
7.259 \\
7.810 \\
8.434\end{array}$ & $\begin{array}{l}18.995 \\
20.310 \\
21.778 \\
23 \cdot 431 \\
25.304\end{array}$ & $\begin{array}{l}1.62 \\
1.64 \\
1.66 \\
1.68 \\
1.70\end{array}$ & $\begin{array}{r}.547 \\
.542 \\
.538 \\
.533 \\
.528\end{array}$ & $\begin{array}{l}.251 \\
: 256 \\
.251 \\
: 266 \\
: 270\end{array}$ & $\begin{array}{r}.371 \\
.387 \\
.402 \\
.419 \\
.435\end{array}$ & $\begin{array}{l}1.486 \\
1.548 \\
1.611 \\
1.676 \\
1.741\end{array}$ \\
\hline & $\begin{array}{r}.093 \\
.087 \\
.080 \\
.073 \\
.066\end{array}$ & $\begin{array}{l}.474 \\
.476 \\
.478 \\
.480 \\
.482\end{array}$ & $\begin{array}{r}9.148 \\
9.971 \\
10.933 \\
12.068 \\
13.431\end{array}$ & $\begin{array}{l}27 \cdot 445 \\
29 \cdot 915 \\
32 \cdot 799 \\
36 \cdot 205 \\
40.294\end{array}$ & $\begin{array}{l}1.72 \\
1: 74 \\
1: 76 \\
1.78 \\
1.80\end{array}$ & $\begin{array}{r}.523 \\
.519 \\
.514 \\
.509 \\
.505\end{array}$ & $\begin{array}{r}.275 \\
.279 \\
.284 \\
.288 \\
.292\end{array}$ & $\begin{array}{l}.452 \\
.468 \\
.486 \\
.503 \\
.521\end{array}$ & $\begin{array}{l}1.808 \\
1.875 \\
1.944 \\
2.015 \\
2.087\end{array}$ \\
\hline & $\begin{array}{l}.060 \\
.053 \\
.046 \\
.040 \\
.033\end{array}$ & $\begin{array}{l}.483 \\
.485 \\
.487 \\
.489 \\
.491\end{array}$ & $\begin{array}{l}15.097 \\
17.180 \\
19.858 \\
23.429\end{array}$ & $\begin{array}{l}45 \cdot 291 \\
51 \cdot 540 \\
59: 576 \\
70 \cdot 287 \\
85 \cdot 269\end{array}$ & $\begin{array}{l}1.82 \\
1.84 \\
1.86 \\
1.88 \\
1.90\end{array}$ & $\begin{array}{r}.500 \\
.495 \\
.490 \\
.486 \\
.481\end{array}$ & $\begin{array}{r}\cdot 296 \\
: 300 \\
: 304 \\
: 308 \\
: 312\end{array}$ & $\begin{array}{r}.540 \\
.558 \\
.577 \\
.597 \\
.616\end{array}$ & $\begin{array}{l}2 \cdot 160 \\
2 \cdot 234 \\
2 \cdot 310 \\
2 \cdot 388 \\
2 \cdot 466\end{array}$ \\
\hline 98 & $\begin{array}{r}.026 \\
.020 \\
.013 \\
.006 \\
0.000\end{array}$ & $\begin{array}{l}.493 \\
.494 \\
.496 \\
.498 \\
.500\end{array}$ & $\begin{array}{l}35 \cdot 9 \\
49: 4 \\
73: 3 \\
99: 9 \\
99 \cdot 9\end{array}$ & $\begin{array}{l}99 \cdot 999 \\
99 \cdot 999 \\
99.999 \\
99 \cdot 999 \\
99.999\end{array}$ & $\begin{array}{l}1.92 \\
1: 94 \\
1: 96 \\
1.98 \\
2.00\end{array}$ & $\begin{array}{r}.476 \\
.472 \\
.467 \\
.462 \\
.457\end{array}$ & $\begin{array}{r}.316 \\
: 319 \\
: 323 \\
: 327 \\
\cdot 330\end{array}$ & $\begin{array}{l}.636 \\
.657 \\
.678 \\
.699 \\
.721\end{array}$ & $\begin{array}{l}2.629 \\
2.713 \\
2.798\end{array}$ \\
\hline
\end{tabular}


$F C=4.0$

$F C=4.0$

\begin{tabular}{|c|c|c|c|c|c|c|c|c|c|}
\hline$=T$ & PHI & PS! & QI & $Q 2$ & $F T$ & $\mathrm{PHI}$ & PSI & $Q_{1}$ & $Q 2$ \\
\hline $\begin{array}{l}.04 \\
.06 \\
08 \\
10 \\
10\end{array}$ & $\begin{array}{r}.453 \\
.448 \\
.443 \\
.439 \\
.434\end{array}$ & $\begin{array}{r}.333 \\
: 337 \\
: 340 \\
.343 \\
.346\end{array}$ & $\begin{array}{l}.743 \\
.766 \\
.789 \\
.813 \\
.837\end{array}$ & $\begin{array}{l}2.975 \\
3.065 \\
3 \cdot 158 \\
3.253 \\
3.350\end{array}$ & $\begin{array}{l}3.02 \\
3.04 \\
3.06 \\
3.08 \\
3.10\end{array}$ & $\begin{array}{l}.221 \\
.216 \\
.21 \\
.211 \\
.207 \\
.202\end{array}$ & $\begin{array}{r}.445 \\
.446 \\
.448 \\
.449 \\
.451\end{array}$ & $\begin{array}{l}3 . \\
3: \\
3: \\
3: \\
3 .\end{array}$ & $\begin{array}{l}12 \cdot 165 \\
12.546 \\
12.943 \\
13.358 \\
13.791\end{array}$ \\
\hline $\begin{array}{l}12 \\
14 \\
16 \\
18 \\
20\end{array}$ & $\begin{array}{r}.429 \\
.425 \\
.420 \\
.415 \\
.411\end{array}$ & $\begin{array}{r}.349 \\
.352 \\
.355 \\
: 358 \\
.361\end{array}$ & $\begin{array}{r}.862 \\
.887 \\
.913 \\
.939 \\
.966\end{array}$ & $\begin{array}{l}3.449 \\
3.550 \\
3.653 \\
3.759 \\
3.867\end{array}$ & $\begin{array}{l}3: 12 \\
3: 14 \\
3: 16 \\
3: 16 \\
3.20\end{array}$ & $\begin{array}{r}198 \\
1193 \\
1 \\
1189 \\
1184 \\
.179\end{array}$ & $\begin{array}{l}.452 \\
.453 \\
.455 \\
.456 \\
.457\end{array}$ & $\begin{array}{l}3.561 \\
3.679 \\
3.803 \\
3.934 \\
4.070\end{array}$ & $\begin{array}{l}14.244 \\
14.718 \\
15.215 \\
15.736 \\
16.283\end{array}$ \\
\hline $\begin{array}{l}22 \\
24 \\
26 \\
28 \\
30\end{array}$ & $\begin{array}{r}.406 \\
: 401 \\
: 397 \\
: 392 \\
.387\end{array}$ & $\begin{array}{r}\cdot 364 \\
: 366 \\
: 369 \\
: 372 \\
\cdot 374\end{array}$ & $\begin{array}{r}\cdot 994 \\
1: 022 \\
1: 051 \\
1: 081 \\
1.111\end{array}$ & $\begin{array}{l}3.978 \\
4.091 \\
4.207 \\
4.325 \\
4.446\end{array}$ & $\begin{array}{l}3 \cdot 22 \\
3 \cdot 24 \\
3 \cdot 26 \\
3 \cdot 28 \\
3.30\end{array}$ & $\begin{array}{l}175 \\
: 170 \\
: 166 \\
: 161 \\
.157\end{array}$ & $\begin{array}{l}.459 \\
.460 \\
.461 \\
.463 \\
.464\end{array}$ & $\begin{array}{l}4 \cdot 214 \\
4 \cdot 366 \\
4 \cdot 525 \\
4 \cdot 694 \\
4 \cdot 872\end{array}$ & $\begin{array}{l}16.858 \\
17.464 \\
18.103 \\
18.777 \\
19.490\end{array}$ \\
\hline $\begin{array}{l}32 \\
34 \\
36 \\
38 \\
40\end{array}$ & $\begin{array}{r}\cdot 383 \\
\cdot 378 \\
: 373 \\
: 369 \\
\cdot 364\end{array}$ & $\begin{array}{r}.377 \\
: 379 \\
: 382 \\
: 384 \\
\cdot 387\end{array}$ & $\begin{array}{l}1 \cdot 142 \\
1 \cdot 174 \\
1 \cdot 207 \\
1.240 \\
1.274\end{array}$ & $\begin{array}{l}4.571 \\
4.698 \\
4.828 \\
4.962 \\
5.099\end{array}$ & $\begin{array}{l}3 \cdot 32 \\
3 \cdot 34 \\
3 \cdot 36 \\
3 \cdot 38 \\
3 \cdot 40\end{array}$ & $\begin{array}{r}.152 \\
: 148 \\
: 143 \\
: 139 \\
.134\end{array}$ & $\begin{array}{l}.465 \\
.466 \\
.468 \\
.469 \\
.470\end{array}$ & $\begin{array}{l}5 \\
5 \\
5 \\
5 \\
5\end{array}$ & $\begin{array}{l}20.244 \\
21.045 \\
21.895 \\
22.801 \\
23.767\end{array}$ \\
\hline $\begin{array}{l}42 \\
44 \\
46 \\
48 \\
50\end{array}$ & $\begin{array}{r}.359 \\
.355 \\
.350 \\
.345 \\
\cdot 341\end{array}$ & $\begin{array}{r}389 \\
: 391 \\
: 394 \\
: 396 \\
\cdot 398\end{array}$ & $\begin{array}{l}1 \cdot 310 \\
1: 346 \\
1: 383 \\
1: 421 \\
1.460\end{array}$ & $\begin{array}{l}5.240 \\
5.384 \\
5.532 \\
5.685 \\
5.841\end{array}$ & $\begin{array}{l}3.42 \\
3.44 \\
3.46 \\
3.48 \\
3.50\end{array}$ & 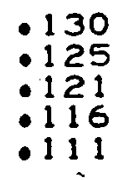 & $\begin{array}{l}.471 \\
.472 \\
.473 \\
.474 \\
.476\end{array}$ & $\begin{array}{l}6.200 \\
6.476 \\
6.774 \\
7.094 \\
7.440\end{array}$ & $\begin{array}{l}24 \cdot 800 \\
25: 907 \\
27: 096 \\
28: 376 \\
29 \cdot 760\end{array}$ \\
\hline $\begin{array}{l}52 \\
54 \\
56 \\
58 \\
60\end{array}$ & $\begin{array}{r}\cdot 336 \\
: 331 \\
-327 \\
\cdot 322 \\
\cdot 317\end{array}$ & $\begin{array}{l}.400 \\
.402 \\
.404 \\
.406 \\
.408\end{array}$ & $\begin{array}{l}1.500 \\
1.541 \\
1.584 \\
1.627 \\
1.672\end{array}$ & $\begin{array}{l}6.001 \\
6 \cdot 166 \\
6 \cdot 336 \\
6.510 \\
6.589\end{array}$ & $\begin{array}{l}3 \cdot 52 \\
3: 54 \\
3: 56 \\
3: 58 \\
3.60\end{array}$ & 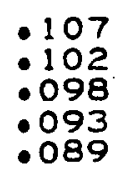 & $\begin{array}{r}.477 \\
.478 \\
.479 \\
.480 \\
.481\end{array}$ & $\begin{array}{l}7.814 \\
8.221 \\
8.666 \\
9.152 \\
9.687\end{array}$ & $\begin{array}{l}31 \cdot 258 \\
32 \cdot 887 \\
34 \cdot 664 \\
36 \cdot 610 \\
38 \cdot 751\end{array}$ \\
\hline $\begin{array}{l}62 \\
64 \\
66 \\
68 \\
70\end{array}$ & $\begin{array}{r}\cdot 313 \\
\cdot 308 \\
: 303 \\
\cdot 299 \\
\cdot 294\end{array}$ & $\begin{array}{r}.410 \\
.412 \\
.414 \\
.416 \\
.418\end{array}$ & $\begin{array}{l}1.718 \\
1.766 \\
1.815 \\
1.865 \\
1.917\end{array}$ & $\begin{array}{r}6.874 \\
7.064 \\
7.260 \\
7.462 \\
7.670\end{array}$ & $\begin{array}{l}3.62 \\
3.64 \\
3.66 \\
3.68 \\
3.70\end{array}$ & $\begin{array}{l}.084 \\
.080 \\
.075 \\
.071 \\
.066\end{array}$ & $\begin{array}{l}.482 \\
.483 \\
.484 \\
.485 \\
.486\end{array}$ & $\begin{array}{l}10.279 \\
10.937 \\
11.671 \\
12.498 \\
13.435\end{array}$ & $\begin{array}{l}41.118 \\
43.748 \\
46.687 \\
49.995 \\
53.742\end{array}$ \\
\hline $\begin{array}{l}74 \\
76 \\
78 \\
80\end{array}$ & $\begin{array}{r}.290 \\
.285 \\
.280 \\
.276 \\
.271\end{array}$ & $\begin{array}{r}.420 \\
.422 \\
.424 \\
.425 \\
.427\end{array}$ & $\begin{array}{l}1 \cdot 971 \\
2 \cdot 026 \\
2 \cdot 083 \\
2 \cdot 142 \\
2 \cdot 203\end{array}$ & $\begin{array}{l}7.885 \\
8.106 \\
8.335 \\
8.571 \\
8.815\end{array}$ & $\begin{array}{l}3.72 \\
3 \cdot 74 \\
3.76 \\
3.78 \\
3.80\end{array}$ & $\begin{array}{l}.062 \\
.058 \\
.053 \\
.049 \\
.044\end{array}$ & $\begin{array}{r}.487 \\
.488 \\
.489 \\
.490 \\
.491\end{array}$ & $\begin{array}{l}14.507 \\
15.743 \\
17.184 \\
18.887 \\
20.932\end{array}$ & $\begin{array}{l}58 \cdot 028 \\
62.972 \\
68 \cdot 738 \\
75.550 \\
83.731\end{array}$ \\
\hline $\begin{array}{l}84 \\
86 \\
88\end{array}$ & $\begin{array}{l}.267 \\
.262 \\
.257 \\
.253 \\
.248\end{array}$ & $\begin{array}{r}.429 \\
.431 \\
.432 \\
.434\end{array}$ & $\begin{array}{l}2 \cdot 267 \\
2 \cdot 332 \\
2.400 \\
2 \cdot 470\end{array}$ & $\begin{array}{r}9.068 \\
9.329 \\
9.600 \\
9.880 \\
10.171\end{array}$ & $\begin{array}{l}3.82 \\
3.84 \\
3.86 \\
3.88\end{array}$ & $\begin{array}{l}.040 \\
.035 \\
.031 \\
.026 \\
.022\end{array}$ & $\begin{array}{r}.492 \\
.493 \\
.493 \\
.494 \\
.495\end{array}$ & $\begin{array}{l}23.431 \\
26.555 \\
30.574 \\
35.928 \\
43.413\end{array}$ & $\begin{array}{l}99 \cdot 9 \\
99 \cdot 9 \\
99 \cdot 9 \\
99 \cdot 9\end{array}$ \\
\hline & $\begin{array}{r}.244 \\
: 239 \\
: 234 \\
: 230 \\
.225\end{array}$ & $\begin{array}{l}.437 \\
.439 \\
.440 \\
.442 \\
.443\end{array}$ & $\begin{array}{l}2.618 \\
2.696 \\
2.777 \\
2.862 \\
2.949\end{array}$ & $\begin{array}{l}10.472 \\
10.785 \\
11.110 \\
11.448 \\
11.799\end{array}$ & $\begin{array}{l}3: \\
3: \\
3: \\
3:\end{array}$ & $\begin{array}{l}.017 \\
.013 \\
0008 \\
0004 \\
0.000\end{array}$ & $\begin{array}{r}.496 \\
.497 \\
: 498 \\
.499 \\
.500\end{array}$ & $\begin{array}{l}54 \cdot 651 \\
73 \cdot 432 \\
99.999 \\
99.999 \\
99.999\end{array}$ & $\begin{array}{l}99 \\
99 \\
99\end{array}$ \\
\hline
\end{tabular}


$F C=5.0$

$F C=5.0$

\begin{tabular}{|c|c|c|c|c|c|c|c|c|c|}
\hline$F T$ & PHI & PS 1 & Q1 & Q2 & $F T$ & $\mathrm{PHI}$ & PSI & Q1 & Q2 \\
\hline $\begin{array}{l}1.02 \\
1: 04 \\
1: 06 \\
1: 08 \\
1.10\end{array}$ & $\begin{array}{l}.689 \\
.686 \\
.682 \\
.679 \\
.675\end{array}$ & $\begin{array}{l}.011 \\
.023 \\
.034 \\
.034 \\
.044 \\
.054\end{array}$ & $\begin{array}{l}.006 \\
.014 \\
.014 \\
.021 \\
.028 \\
.035\end{array}$ & $\begin{array}{r}.034 \\
.070 \\
.105 \\
.1142 \\
.178\end{array}$ & $\begin{array}{l}2.02 \\
2.04 \\
2.06 \\
2.08 \\
2.10\end{array}$ & $\begin{array}{l}.513 \\
.509 \\
.505 \\
.502 \\
.498\end{array}$ & $\begin{array}{r}.312 \\
.315 \\
.318 \\
.321 \\
.321 \\
.324\end{array}$ & $\begin{array}{r}.491 \\
.504 \\
.518 \\
.531 \\
.545\end{array}$ & $\begin{array}{l}2.458 \\
2 \cdot 524 \\
2.591 \\
2.559 \\
2.729\end{array}$ \\
\hline 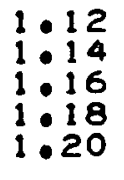 & $\begin{array}{l}.672 \\
.668 \\
.665 \\
.661 \\
.658\end{array}$ & $\begin{array}{l}.064 \\
.074 \\
.083 \\
.083 \\
.092 \\
.101\end{array}$ & $\begin{array}{l}.043 \\
.050 \\
.058 \\
.065 \\
.073\end{array}$ & $\begin{array}{r}.215 \\
.253 \\
.291 \\
.229 \\
.368\end{array}$ & $\begin{array}{l}2: 12 \\
2: 14 \\
2: 16 \\
2: 18 \\
2.20\end{array}$ & $\begin{array}{l}.495 \\
.491 \\
.488 \\
.484 \\
.481\end{array}$ & $\begin{array}{r}\cdot 327 \\
: 329 \\
\cdot 332 \\
\cdot 335 \\
\cdot 337\end{array}$ & $\begin{array}{r}.559 \\
.574 \\
.588 \\
.603 \\
.617\end{array}$ & $\begin{array}{l}2.799 \\
2: 870 \\
2: 942 \\
3.015 \\
3.089\end{array}$ \\
\hline $\begin{array}{l}1 \cdot 22 \\
1: 24 \\
1: 26 \\
1: 28 \\
1.30\end{array}$ & $\begin{array}{l}.654 \\
.651 \\
.647 \\
.644 \\
.640\end{array}$ & 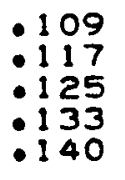 & $\begin{array}{l}.081 \\
.089 \\
.097 \\
.095 \\
.1105 \\
.114\end{array}$ & $\begin{array}{l}.407 \\
.447 \\
.488 \\
.528 \\
.570\end{array}$ & $\begin{array}{l}2 \cdot 22 \\
2 \cdot 24 \\
2 \cdot 26 \\
2 \cdot 28 \\
2 \cdot 30\end{array}$ & $\begin{array}{l}.477 \\
.474 \\
.470 \\
.467 \\
.463\end{array}$ & $\begin{array}{r}.340 \\
.343 \\
.345 \\
\cdot 348 \\
\cdot 350\end{array}$ & $\begin{array}{l}.633 \\
.648 \\
.663 \\
.679 \\
.695\end{array}$ & $\begin{array}{l}3 \cdot 165 \\
3 \cdot 241 \\
3 \cdot 319 \\
3.398 \\
3.478\end{array}$ \\
\hline $\begin{array}{l}1 \cdot 32 \\
1: 34 \\
1: 36 \\
1: 38 \\
1: 40\end{array}$ & $\begin{array}{l}.637 \\
.633 \\
.630 \\
.626 \\
.622\end{array}$ & 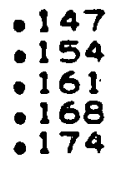 & $\begin{array}{r}122 \\
1130 \\
1139 \\
1148 \\
1156\end{array}$ & $\begin{array}{l}.612 \\
.654 \\
.697 \\
.740 \\
.784\end{array}$ & $\begin{array}{l}2 \cdot 32 \\
2: 34 \\
2: 36 \\
2: 38 \\
2 \cdot 40\end{array}$ & $\begin{array}{l}.460 \\
.456 \\
.452 \\
.449 \\
.445\end{array}$ & $\begin{array}{l}\cdot 352 \\
: 355 \\
: 357 \\
\cdot 359 \\
\cdot 362\end{array}$ & $\begin{array}{l}.711 \\
.728 \\
.745 \\
.762 \\
.772\end{array}$ & $\begin{array}{l}3.559 \\
3.641 \\
3.725 \\
3.810 \\
3.897\end{array}$ \\
\hline $\begin{array}{l}1.42 \\
1.44 \\
1.46 \\
1.48 \\
1.50\end{array}$ & $\begin{array}{l}.619 \\
.615 \\
.612 \\
.608 \\
.605\end{array}$ & $\begin{array}{r}180 \\
1186 \\
1192 \\
1198 \\
.204\end{array}$ & $\begin{array}{r}1165 \\
1174 \\
1183 \\
1193 \\
.1202\end{array}$ & $\begin{array}{r}.828 \\
.873 \\
.919 \\
.965 \\
1.011\end{array}$ & $\begin{array}{l}2.42 \\
2.44 \\
2.46 \\
2.48 \\
2.50\end{array}$ & $\begin{array}{r}.442 \\
.438 \\
.435 \\
.431 \\
.428\end{array}$ & $\begin{array}{r}.364 \\
.366 \\
.368 \\
.370 \\
.372\end{array}$ & $\begin{array}{r}.797 \\
.814 \\
8332 \\
.851 \\
.870\end{array}$ & $\begin{array}{l}3 \cdot 985 \\
4 \cdot 074 \\
4 \cdot 164 \\
4 \cdot 256 \\
4 \cdot 350\end{array}$ \\
\hline $\begin{array}{l}1.52 \\
1: 54 \\
1: 56 \\
1: 58 \\
1.50\end{array}$ & $\begin{array}{r}.601 \\
.598 \\
.594 \\
.591 \\
.587\end{array}$ & $\begin{array}{l}.209 \\
.214 \\
.220 \\
.225 \\
.230\end{array}$ & $\begin{array}{l}.211 \\
: 221 \\
.221 \\
2231 \\
.240 \\
.250\end{array}$ & $\begin{array}{l}1.059 \\
1.106 \\
1.155 \\
1.204 \\
1.253\end{array}$ & $\begin{array}{l}2 \cdot 52 \\
2: 54 \\
2: 56 \\
2: 58 \\
2 \cdot 60\end{array}$ & $\begin{array}{r}.424 \\
.421 \\
.417 \\
.414 \\
.410\end{array}$ & $\begin{array}{r}\cdot 374 \\
: 376 \\
\cdot 378 \\
\cdot 380 \\
\cdot 382\end{array}$ & $\begin{array}{r}.889 \\
.908 \\
.928 \\
.948 \\
.968\end{array}$ & $\begin{array}{l}4.445 \\
4.542 \\
4.640 \\
4.740 \\
4.842\end{array}$ \\
\hline $\begin{array}{l}1.62 \\
1.64 \\
1.66 \\
1.68 \\
1.70\end{array}$ & $\begin{array}{r}.583 \\
.580 \\
.576 \\
.573 \\
.569\end{array}$ & $\begin{array}{r}.235 \\
.239 \\
.244 \\
.248 \\
.253\end{array}$ & $\begin{array}{l}.260 \\
.270 \\
.281 \\
.291 \\
.292\end{array}$ & $\begin{array}{l}1.304 \\
1.354 \\
1.406 \\
1.458 \\
1.511\end{array}$ & $\begin{array}{l}2.62 \\
2 \cdot 64 \\
2.66 \\
2 \cdot 68 \\
2.70\end{array}$ & $\begin{array}{l}.407 \\
.403 \\
.400 \\
.396 \\
.393\end{array}$ & $\begin{array}{l}\cdot 384 \\
.386 \\
.388 \\
\cdot 389 \\
.391\end{array}$ & $\begin{array}{l}.989 \\
1.010 \\
1.031 \\
1.053 \\
1.075\end{array}$ & $\begin{array}{l}4 \cdot 945 \\
5 \cdot 051 \\
5 \cdot 158 \\
5 \cdot 267 \\
5 \cdot 377\end{array}$ \\
\hline $\begin{array}{l}1: 72 \\
1: 74 \\
1: 76 \\
1: 78 \\
1: 80\end{array}$ & $\begin{array}{l}.566 \\
.562 \\
.559 \\
.555 \\
.552\end{array}$ & $\begin{array}{l}.257 \\
.261 \\
.265 \\
.269 \\
.273\end{array}$ & $\begin{array}{r}.313 \\
.323 \\
.334 \\
.345 \\
.357\end{array}$ & $\begin{array}{l}1.565 \\
1.619 \\
1.674 \\
1.729 \\
1.786\end{array}$ & $\begin{array}{l}2 \cdot 72 \\
2: 74 \\
2: 76 \\
2: 78 \\
2 \cdot 80\end{array}$ & $\begin{array}{r}\cdot 389 \\
\cdot 386 \\
\cdot 382 \\
\cdot 379 \\
\cdot 375\end{array}$ & $\begin{array}{l}.393 \\
.395 \\
.396 \\
.398 \\
.400\end{array}$ & $\begin{array}{l}1.098 \\
1.121 \\
1.144 \\
1.168 \\
1.192\end{array}$ & $\begin{array}{l}5.490 \\
5.605 \\
5.722 \\
5.841 \\
5.962\end{array}$ \\
\hline $\begin{array}{l}1.82 \\
1: 84 \\
1.86 \\
1.88 \\
1.90\end{array}$ & $\begin{array}{r}.548 \\
.544 \\
.544 \\
.541 \\
.537 \\
.534\end{array}$ & $\begin{array}{r}.277 \\
.281 \\
.285 \\
.288 \\
.292\end{array}$ & $\begin{array}{r}.368 \\
.380 \\
.391 \\
.403 \\
.415\end{array}$ & $\begin{array}{l}1.843 \\
1.901 \\
1.959 \\
2.0119 \\
2.079\end{array}$ & $\begin{array}{l}2.82 \\
2.84 \\
2.86 \\
2.88 \\
2 \cdot 90\end{array}$ & $\begin{array}{l}\cdot 372 \\
.368 \\
.365 \\
.361 \\
.358\end{array}$ & $\begin{array}{l}.401 \\
.403 \\
.404 \\
.406 \\
.407\end{array}$ & $\begin{array}{l}1.217 \\
1.242 \\
1.268 \\
1.294 \\
1.320\end{array}$ & $\begin{array}{l}6.086 \\
6.212 \\
6.340 \\
6.471 \\
6.604\end{array}$ \\
\hline $\begin{array}{l}1.92 \\
1: 94 \\
1.96 \\
1.98 \\
2.00\end{array}$ & $\begin{array}{r}.530 \\
.527 \\
.523 \\
.520 \\
.516\end{array}$ & $\begin{array}{r}.295 \\
.299 \\
.302 \\
.305 \\
.309\end{array}$ & $\begin{array}{l}.428 \\
.440 \\
.452 \\
.465 \\
.478\end{array}$ & $\begin{array}{l}2 \cdot 140 \\
2 \cdot 202 \\
2 \cdot 264 \\
2 \cdot 328 \\
2 \cdot 392\end{array}$ & $\begin{array}{l}2 \cdot 92 \\
2: 94 \\
2 \cdot 96 \\
2 \cdot 98 \\
3.00\end{array}$ & $\begin{array}{l}\cdot 354 \\
\cdot 351 \\
\cdot 347 \\
\cdot 344 \\
\cdot 340\end{array}$ & $\begin{array}{r}.409 \\
.410 \\
.412 \\
.413 \\
.415\end{array}$ & $\begin{array}{l}1.348 \\
1.375 \\
1.404 \\
1.432 \\
1.462\end{array}$ & $\begin{array}{l}6.740 \\
6.878 \\
7.020 \\
7.164 \\
7.311\end{array}$ \\
\hline
\end{tabular}


$F C=5.0$

$F C=5.0$

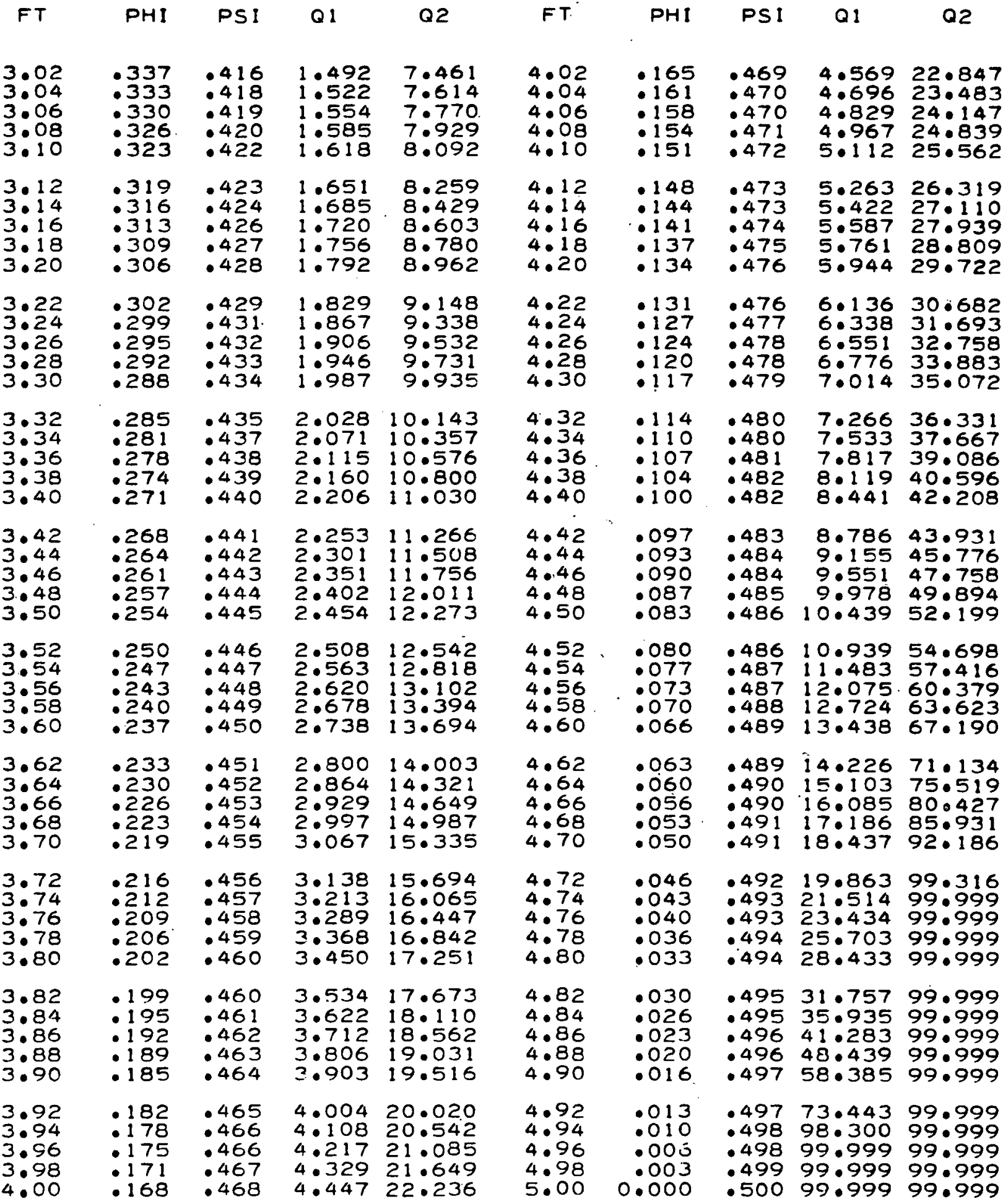


$F C=8.0$

$F C=8.0$

\begin{tabular}{|c|c|c|c|c|c|c|c|c|c|}
\hline$F T$ & PHI & PSI & 01 & Q2 & $F T$ & PHI & PSI & 01 & Q2 \\
\hline $\begin{array}{l}1.02 \\
1: 04 \\
1: 06 \\
1: 08 \\
1.010\end{array}$ & $\begin{array}{l}.691 \\
.689 \\
.687 \\
.685 \\
.683\end{array}$ & $\begin{array}{r}.010 \\
.021 \\
.031 \\
.031 \\
.040 \\
.050\end{array}$ & $\begin{array}{r}.003 \\
.007 \\
.012 \\
.012 \\
.016 \\
.020\end{array}$ & $\begin{array}{r}.031 \\
.063 \\
.096 \\
.092 \\
.161\end{array}$ & $\begin{array}{l}2.02 \\
2.04 \\
2.06 \\
2.08 \\
2.10\end{array}$ & $\begin{array}{r}.590 \\
.588 \\
.586 \\
.584 \\
.582\end{array}$ & $\begin{array}{r}.284 \\
.287 \\
.289 \\
.292 \\
.295\end{array}$ & $\begin{array}{l}.243 \\
.249 \\
.254 \\
.260 \\
.266\end{array}$ & $\begin{array}{l}1 \cdot 947 \\
1 \cdot 992 \\
2 \cdot 038 \\
2 \cdot 084 \\
2 \cdot 131\end{array}$ \\
\hline $\begin{array}{l}1: 12 \\
1: 14 \\
1: 16 \\
1: 18 \\
1: 20\end{array}$ & $\begin{array}{l}.681 \\
.679 \\
.677 \\
.675 \\
.673\end{array}$ & $\begin{array}{l}.059 \\
.067 \\
.076 \\
.084 \\
.092\end{array}$ & $\begin{array}{l}.024 \\
.028 \\
.032 \\
.036 \\
.041\end{array}$ & $\begin{array}{r}.194 \\
.227 \\
.261 \\
.295 \\
.329\end{array}$ & $\begin{array}{l}2 \cdot 12 \\
2: 14 \\
2: 16 \\
2: 18 \\
2.20\end{array}$ & $\begin{array}{l}.579 \\
.577 \\
.575 \\
.573 \\
.571\end{array}$ & $\begin{array}{r}.297 \\
.300 \\
.302 \\
.305 \\
.307\end{array}$ & $\begin{array}{r}.272 \\
.278 \\
.284 \\
.290 \\
.296\end{array}$ & $\begin{array}{l}2 \cdot 178 \\
2 \cdot 225 \\
2 \cdot 272 \\
2 \cdot 320 \\
2 \cdot 368\end{array}$ \\
\hline $\begin{array}{l}1 \cdot 22 \\
1: 24 \\
1: 26 \\
1: 28 \\
1: 30\end{array}$ & $\begin{array}{l}.671 \\
.669 \\
.667 \\
.665 \\
.663\end{array}$ & $\begin{array}{r}.099 \\
.1107 \\
1114 \\
1121 \\
1128\end{array}$ & $\begin{array}{l}.045 \\
.049 \\
.054 \\
.058 \\
.062\end{array}$ & $\begin{array}{r}.363 \\
.397 \\
.432 \\
.467 \\
.502\end{array}$ & $\begin{array}{l}2 \cdot 22 \\
2: 24 \\
2: 26 \\
2: 28 \\
2 \cdot 30\end{array}$ & $\begin{array}{l}.569 \\
.567 \\
.565 \\
.563 \\
.561\end{array}$ & $\begin{array}{r}.310 \\
.312 \\
.314 \\
.314 \\
.317 \\
.319\end{array}$ & $\begin{array}{r}.302 \\
.308 \\
.314 \\
.320 \\
\cdot 326\end{array}$ & $\begin{array}{l}2.417 \\
2.466 \\
2 \cdot 515 \\
2 \cdot 565 \\
2.615\end{array}$ \\
\hline $\begin{array}{l}1 \cdot 32 \\
1: 34 \\
1: 36 \\
1: 38 \\
1: 40\end{array}$ & $\begin{array}{l}.661 \\
.659 \\
.657 \\
.655 \\
.653\end{array}$ & 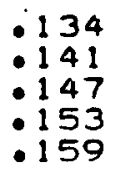 & $\begin{array}{l}.067 \\
.071 \\
.076 \\
.080 \\
.085\end{array}$ & $\begin{array}{l}.538 \\
.573 \\
.609 \\
.646 \\
.682\end{array}$ & $\begin{array}{l}2 \cdot 32 \\
2: 34 \\
2 \cdot 36 \\
2: 38 \\
2 \cdot 40\end{array}$ & $\begin{array}{l}.559 \\
.557 \\
.555 \\
.553 \\
.551\end{array}$ & $\begin{array}{r}.321 \\
.323 \\
.325 \\
.327 \\
.329\end{array}$ & $\begin{array}{r}.333 \\
.339 \\
.345 \\
.352 \\
.358\end{array}$ & $\begin{array}{l}2 \cdot 665 \\
2 \cdot 716 \\
2 \cdot 767 \\
2 \cdot 818 \\
2.870\end{array}$ \\
\hline $\begin{array}{l}1.42 \\
1.44 \\
1.46 \\
1.48 \\
1.50\end{array}$ & $\begin{array}{l}.651 \\
.649 \\
.646 \\
.644 \\
.642\end{array}$ & $\begin{array}{r}1164 \\
1170 \\
1175 \\
1181 \\
1186\end{array}$ & $\begin{array}{l}.089 \\
.094 \\
.099 \\
.103 \\
.108\end{array}$ & $\begin{array}{r}.719 \\
.756 \\
.793 \\
.830 \\
.868\end{array}$ & $\begin{array}{l}2.42 \\
2.44 \\
2.46 \\
2.48 \\
2.50\end{array}$ & $\begin{array}{l}.549 \\
.547 \\
.545 \\
.543 \\
.541\end{array}$ & $\begin{array}{r}.331 \\
.333 \\
.335 \\
.337 \\
.339\end{array}$ & $\begin{array}{r}\cdot 365 \\
: 371 \\
: 378 \\
: 385 \\
\cdot 391\end{array}$ & $\begin{array}{l}2 \cdot 922 \\
2 \cdot 975 \\
3 \cdot 028 \\
3 \cdot 081 \\
3 \cdot 135\end{array}$ \\
\hline $\begin{array}{l}1.52 \\
1.54 \\
1.56 \\
1.58 \\
1.60\end{array}$ & $\begin{array}{l}.640 \\
.638 \\
.636 \\
.634 \\
.632\end{array}$ & $\begin{array}{r}191 \\
.196 \\
.200 \\
.205 \\
.209\end{array}$ & 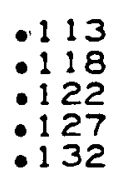 & $\begin{array}{r}.906 \\
.944 \\
.983 \\
1.022 \\
1.061\end{array}$ & $\begin{array}{l}2 \cdot 52 \\
2 \cdot 54 \\
2 \cdot 56 \\
2 \cdot 58 \\
2 \cdot 60\end{array}$ & $\begin{array}{r}.539 \\
.537 \\
.535 \\
.533 \\
.531\end{array}$ & $\begin{array}{r}.341 \\
.343 \\
.344 \\
.346 \\
\cdot 348\end{array}$ & $\begin{array}{r}.398 \\
.405 \\
.412 \\
.419 \\
.426\end{array}$ & $\begin{array}{l}3 \cdot 189 \\
3.244 \\
3 \cdot 299 \\
3 \cdot 355 \\
3.411\end{array}$ \\
\hline $\begin{array}{l}1.62 \\
1.64 \\
1.66 \\
1.68 \\
1.70\end{array}$ & $\begin{array}{l}.630 \\
.628 \\
.626 \\
.624 \\
.622\end{array}$ & $\begin{array}{r}.214 \\
.218 \\
.222 \\
.226 \\
.230\end{array}$ & 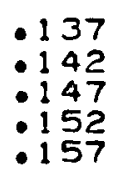 & $\begin{array}{l}1.100 \\
1: 140 \\
1: 180 \\
1: 220 \\
1.260\end{array}$ & $\begin{array}{l}2.62 \\
2.64 \\
2.66 \\
2.68 \\
2.70\end{array}$ & $\begin{array}{r}.529 \\
.527 \\
.525 \\
.523 \\
.521\end{array}$ & $\begin{array}{r}.350 \\
.351 \\
.353 \\
.355 \\
.356\end{array}$ & $\begin{array}{l}.433 \\
.440 \\
.447 \\
.454 \\
.462\end{array}$ & $\begin{array}{l}3.467 \\
3.524 \\
3.581 \\
3.639 \\
3.697\end{array}$ \\
\hline $\begin{array}{l}1.72 \\
1: 74 \\
1: 76 \\
1: 78 \\
1: 80\end{array}$ & $\begin{array}{r}.620 \\
.618 \\
.616 \\
.614 \\
.612\end{array}$ & $\begin{array}{r}.234 \\
: 238 \\
: 242 \\
: 246 \\
.249\end{array}$ & $\begin{array}{r}1 \\
: 162 \\
1167 \\
1172 \\
: 178 \\
: 183\end{array}$ & $\begin{array}{l}1.301 \\
1.342 \\
1.383 \\
1.425 \\
1.467\end{array}$ & $\begin{array}{l}2.72 \\
2.74 \\
2.76 \\
2.78 \\
2.80\end{array}$ & $\begin{array}{r}.519 \\
.517 \\
.515 \\
.513 \\
.511\end{array}$ & $\begin{array}{r}.358 \\
.359 \\
.361 \\
.363 \\
.364\end{array}$ & $\begin{array}{l}.469 \\
.476 \\
.484 \\
.491 \\
.499\end{array}$ & $\begin{array}{l}3.755 \\
3.814 \\
3.874 \\
3.934 \\
3.994\end{array}$ \\
\hline $\begin{array}{l}1.82 \\
1.84 \\
1.86 \\
1.88 \\
1.90\end{array}$ & $\begin{array}{l}.610 \\
.608 \\
.606 \\
.604 \\
.602\end{array}$ & $\begin{array}{l}.253 \\
.256 \\
.259 \\
.263 \\
.266\end{array}$ & 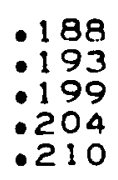 & $\begin{array}{l}1.509 \\
1.551 \\
1.594 \\
1.637 \\
1.680\end{array}$ & $\begin{array}{l}2.82 \\
2.84 \\
2.86 \\
2.88 \\
2.90\end{array}$ & $\begin{array}{r}.508 \\
.506 \\
.504 \\
.502 \\
.500\end{array}$ & $\begin{array}{r}.365 \\
: 367 \\
: 368 \\
: 370 \\
.371\end{array}$ & $\begin{array}{r}.506 \\
.514 \\
.522 \\
.530 \\
.538\end{array}$ & $\begin{array}{l}4.055 \\
4: 116 \\
4: 178 \\
4: 241 \\
4: 304\end{array}$ \\
\hline 1. & $\begin{array}{r}.600 \\
.598 \\
.596 \\
.594 \\
.592\end{array}$ & $\begin{array}{r}.269 \\
.272 \\
.275 \\
.278 \\
.281\end{array}$ & $\begin{array}{l}.215 \\
.221 \\
.2226 \\
.232 \\
.237\end{array}$ & $\begin{array}{l}1.724 \\
1.768 \\
1.812 \\
1.857 \\
1.902\end{array}$ & $\begin{array}{l}2.92 \\
2: 94 \\
2.96 \\
2.98 \\
3.00\end{array}$ & $\begin{array}{l}.498 \\
.496 \\
.494 \\
.492 \\
.490\end{array}$ & $\begin{array}{r}.373 \\
: 374 \\
.375 \\
: 377 \\
.378\end{array}$ & $\begin{array}{r}.545 \\
.553 \\
.561 \\
.570 \\
.578\end{array}$ & $\begin{array}{l}4.367 \\
4.431 \\
4.495 \\
4.560 \\
4.626\end{array}$ \\
\hline
\end{tabular}


$F C=8.0$

$F C=8.0$

\begin{tabular}{|c|c|c|c|c|c|c|c|c|c|}
\hline$F T$ & PHI & PSI & $Q 1$ & Q2 & $F T$ & PHI & PSI & 01 & 02 \\
\hline $\begin{array}{l}3.05 \\
3.10 \\
3.15 \\
3.20 \\
3.25\end{array}$ & $\begin{array}{r}.485 \\
.480 \\
.475 \\
.470 \\
.465\end{array}$ & $\begin{array}{r}\cdot 381 \\
\cdot 384 \\
\cdot 387 \\
\cdot 390 \\
\cdot 393\end{array}$ & $\begin{array}{l}.599 \\
.620 \\
.641 \\
.664 \\
.686\end{array}$ & $\begin{array}{l}4.792 \\
4.961 \\
5.135 \\
5.312 \\
5.492\end{array}$ & $\begin{array}{l}5.55 \\
5.60 \\
5.65 \\
5.70 \\
5.75\end{array}$ & $\begin{array}{r}.236 \\
.231 \\
.227 \\
.222 \\
.217\end{array}$ & $\begin{array}{l}.468 \\
.469 \\
.469 \\
.470 \\
.471\end{array}$ & $\begin{array}{l}2.742 \\
2.831 \\
2.924 \\
3.020 \\
3.121\end{array}$ & $\begin{array}{l}21.940 \\
22.651 \\
23.392 \\
24.166 \\
24.974\end{array}$ \\
\hline $\begin{array}{l}.30 \\
.35 \\
.40 \\
45 \\
.50\end{array}$ & $\begin{array}{l}.460 \\
.455 \\
.450 \\
.445 \\
.440\end{array}$ & $\begin{array}{l}.396 \\
.398 \\
.401 \\
.403 \\
.406\end{array}$ & $\begin{array}{r}.709 \\
.733 \\
.757 \\
.782 \\
.807\end{array}$ & $\begin{array}{l}5.677 \\
5.865 \\
6.058 \\
6.256 \\
6.457\end{array}$ & $\begin{array}{l}5.80 \\
5.85 \\
5.90 \\
5.95 \\
6.00\end{array}$ & $\begin{array}{r}.212 \\
.207 \\
.202 \\
.197 \\
.192\end{array}$ & $\begin{array}{l}.472 \\
.473 \\
.474 \\
.475 \\
.475\end{array}$ & $\begin{array}{l}3 \cdot 227 \\
3 \cdot 338 \\
3.453 \\
3.575 \\
3.703\end{array}$ & $\begin{array}{l}25 \cdot 819 \\
26 \cdot 704 \\
27.631 \\
28.603 \\
29.625\end{array}$ \\
\hline & $\begin{array}{l}.435 \\
.430 \\
.425 \\
.420 \\
.415\end{array}$ & $\begin{array}{r}.408 \\
.410 \\
.413 \\
.415 \\
.417\end{array}$ & $\begin{array}{r}.832 \\
.859 \\
.886 \\
.914 \\
.942\end{array}$ & $\begin{array}{l}6.663 \\
6.874 \\
7.090 \\
7.312 \\
7.538\end{array}$ & $\begin{array}{l}6 \cdot 05 \\
6 \cdot 10 \\
6 \cdot 15 \\
6 \cdot 20 \\
6.25\end{array}$ & $\begin{array}{r}1187 \\
: 183 \\
1178 \\
1173 \\
: 168\end{array}$ & $\begin{array}{l}.476 \\
.477 \\
.478 \\
.479 \\
.479\end{array}$ & $\begin{array}{l}3.837 \\
3.978 \\
4.127 \\
4.284 \\
4.451\end{array}$ & $\begin{array}{l}30.699 \\
31.829 \\
33.020 \\
34.278 \\
35.608\end{array}$ \\
\hline & $\begin{array}{r}.410 \\
.405 \\
.400 \\
.395 \\
.390\end{array}$ & $\begin{array}{r}.419 \\
.421 \\
.423 \\
.425 \\
.427\end{array}$ & $\begin{array}{r}.971 \\
1.000 \\
1.031 \\
1.062 \\
1.094\end{array}$ & $\begin{array}{l}7.770 \\
8.007 \\
8.251 \\
8.500 \\
8.755\end{array}$ & $\begin{array}{l}6 \cdot 30 \\
6.35 \\
6.40 \\
6.45 \\
6.50\end{array}$ & $\begin{array}{r}163 \\
: 158 \\
: 153 \\
: 149 \\
.1\end{array}$ & $\begin{array}{r}.480 \\
.481 \\
.481 \\
.482 \\
.483\end{array}$ & $\begin{array}{l}4.627 \\
4.813 \\
5.012 \\
5.223 \\
5.449\end{array}$ & $\begin{array}{l}37.017 \\
38.511 \\
40.099 \\
41.789 \\
43.593\end{array}$ \\
\hline & $\begin{array}{r}.385 \\
.380 \\
.375 \\
.370 \\
.365\end{array}$ & $\begin{array}{r}.429 \\
.430 \\
.432 \\
.434 \\
.435\end{array}$ & $\begin{array}{l}1.127 \\
1: 161 \\
1: 195 \\
1.231 \\
1.267\end{array}$ & $\begin{array}{r}\dot{9} .019 \\
9.288 \\
9.564 \\
9.848 \\
10.139\end{array}$ & $\begin{array}{l}6.55 \\
6.60 \\
6.65 \\
6.70 \\
6.75\end{array}$ & 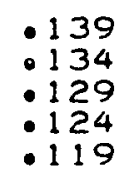 & $\begin{array}{r}.484 \\
.484 \\
.485 \\
.486 \\
.486\end{array}$ & $\begin{array}{l}5.690 \\
5.948 \\
6.225 \\
6.524 \\
6.847\end{array}$ & $\begin{array}{l}45 \cdot 520 \\
47 \cdot 586 \\
49 \cdot 804 \\
52 \cdot 194 \\
54.776\end{array}$ \\
\hline & $\begin{array}{r}.360 \\
: 355 \\
: 350 \\
: 345 \\
.340\end{array}$ & $\begin{array}{r}.437 \\
.439 \\
.440 \\
.442 \\
.443\end{array}$ & $\begin{array}{l}1 \cdot 304 \\
1: 343 \\
1 \cdot 382 \\
1: 423 \\
1 \cdot 465\end{array}$ & $\begin{array}{l}10.439 \\
10.746 \\
11.063 \\
11.388 \\
11.723\end{array}$ & $\begin{array}{l}6.80 \\
6.85 \\
6.9 \\
6.90 \\
7.0\end{array}$ & 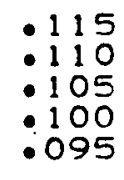 & $\begin{array}{r}.487 \\
.487 \\
.488 \\
.489 \\
.489\end{array}$ & $\begin{array}{l}7.196 \\
7.576 \\
7.991 \\
8.445 \\
8.944\end{array}$ & $\begin{array}{l}57.574 \\
60.614 \\
63.929 \\
67.56 \\
71.559\end{array}$ \\
\hline & $\begin{array}{r}.335 \\
.330 \\
.325 \\
.320 \\
.315\end{array}$ & $\begin{array}{l}.444 \\
.446 \\
.447 \\
.449 \\
.450\end{array}$ & $\begin{array}{r}1.508 \\
1.552 \\
1.598 \\
1.545 \\
1.694\end{array}$ & $\begin{array}{l}12.067 \\
12.422 \\
12.787 \\
13.164 \\
13.552\end{array}$ & $\begin{array}{l}7.05 \\
7: 10 \\
7: 15 \\
7.20 \\
7.25\end{array}$ & $\begin{array}{l}.090 \\
.086 \\
.081 \\
.076 \\
.071\end{array}$ & $\begin{array}{r}.490 \\
.490 \\
.491 \\
.492 \\
.492\end{array}$ & $\begin{array}{r}9.496 \\
10.110 \\
10.796 \\
11.568 \\
12.442\end{array}$ & $\begin{array}{l}75 \cdot 975 \\
80.88 \\
86.372 \\
92.54 \\
99.539\end{array}$ \\
\hline & $\begin{array}{r}.310 \\
.305 \\
.300 \\
.295 \\
.290\end{array}$ & $\begin{array}{l}.451 \\
.452 \\
.454 \\
.455 \\
.456\end{array}$ & $\begin{array}{l}1.744 \\
1.795 \\
1.849 \\
1.904 \\
1.961\end{array}$ & $\begin{array}{l}13.953 \\
14.366 \\
14.793 \\
15.234 \\
15.690\end{array}$ & $\begin{array}{l}7 \cdot 30 \\
7: 35 \\
7: 40 \\
7: 45 \\
7: 50\end{array}$ & $\begin{array}{l}.066 \\
.062 \\
.057 \\
.052 \\
.047\end{array}$ & $\begin{array}{r}.493 \\
.493 \\
.494 \\
.494 \\
.495\end{array}$ & $\begin{array}{l}13.441 \\
14.595 \\
15.940 \\
17.532 \\
19.441\end{array}$ & $\begin{array}{l}99 \cdot 99 \\
99: 99 \\
99: 99 \\
99: 99 \\
99.99\end{array}$ \\
\hline & $\begin{array}{r}.286 \\
.281 \\
.276 \\
.271 \\
.266\end{array}$ & $\begin{array}{r}.457 \\
.458 \\
.459 \\
.460 \\
.462\end{array}$ & $\begin{array}{l}2.020 \\
2.081 \\
2.144 \\
2.209 \\
2.277\end{array}$ & $\begin{array}{l}16.161 \\
16.649 \\
17.153 \\
17.677 \\
18.219\end{array}$ & $\begin{array}{l}7.55 \\
7.60 \\
7.65 \\
7.70\end{array}$ & $\begin{array}{r}.042 \\
.038 \\
.033 \\
.028 \\
.023\end{array}$ & $\begin{array}{l}.495 \\
.496 \\
.496 \\
.497 \\
.497\end{array}$ & $\begin{array}{l}21.769 \\
24.689 \\
28.436 \\
33.433 \\
40.415\end{array}$ & $\begin{array}{l}99.999 \\
99.99 \\
99.995 \\
99.995\end{array}$ \\
\hline & $\begin{array}{l}.261 \\
.256 \\
.251 \\
.246 \\
.241\end{array}$ & $\begin{array}{l}.463 \\
.464 \\
.465 \\
.466 \\
.467\end{array}$ & $\begin{array}{l}2 \cdot 347 \\
2 \cdot 420 \\
2 \cdot 496 \\
2 \cdot 575 \\
2.657\end{array}$ & $\begin{array}{l}18.781 \\
19.365 \\
19.971 \\
2.0 .601 \\
21.257\end{array}$ & $\begin{array}{l}7.80 \\
7.85 \\
7.90 \\
7.95 \\
8.00\end{array}$ & $\begin{array}{l}.019 \\
.014 \\
.009 \\
.004 \\
0.000\end{array}$ & $\begin{array}{r}.498 \\
.498 \\
.499 \\
.499 \\
.500\end{array}$ & $\begin{array}{l}50.893 \\
68 \cdot 342 \\
99 \cdot 999 \\
99 \cdot 999 \\
99.999\end{array}$ & 9 \\
\hline
\end{tabular}




\section{REFERENCES}

1. L. M. Dorfman and M. S. Matheson, "Pulse Radiolysis," Progress in Reaction Kinetics, Vol. 3, G. Porter, Ed., Pergamon Press (1965).

2. J. P. Keene, Pulse Radiolysis, M. Ebert, J. P. Keene, A. J. Swallow, and J. H. Baxendale, Eds., Academic Press (1965). 